\title{
The Excitation of Equatorial Waves by Deep Convection in the NCAR Community Climate Model (CCM3)
}

\author{
LuCREZIA Ricciardulli* AND Rolando R. GARCIA \\ National Center for Atmospheric Research, ${ }^{+}$Boulder, Colorado
}

(Manuscript received 9 July 1999, in final form 9 February 2000)

\begin{abstract}
The forcing of equatorial waves by convective heating in the National Center for Atmospheric Research Community Climate Model (CCM3) is investigated and compared with the forcing deduced from observations of convective clouds. The analysis is performed on two different simulations, wherein convection is represented by the Zhang-McFarlane and the Hack parameterization schemes, respectively. Spectra of equatorial waves excited by convective heating (Rossby, Kelvin, and gravity waves) are obtained by projecting the heating field onto Hough modes; the dynamical response to the heating is then calculated in terms of the vertical component of the Eliassen-Palm flux, $F_{z}$, focusing on waves that are able to propagate into the middle atmosphere. The same analysis is repeated using observations of outgoing longwave radiation as a proxy for tropical convection. Comparison of CCM3 results with those derived from observations indicates that high-frequency heating variability is underestimated in both CCM3 simulations, despite the fact that time-mean values of convective heating are well represented. Moreover, the two convective parameterization schemes differ substantially from each other: Compared to observations, $F_{z}$ is severely underestimated at most frequencies when CCM3 is run with the Zhang-McFarlane scheme. When the Hack scheme is used, $F_{z}$ at frequencies $|\omega|<0.5$ cycles per day is comparable to the observations, but it is underestimated at higher frequencies. Misrepresentation of the variability of convective heating is likely to have important consequences for the dynamical simulation of the middle atmosphere and even the troposphere.
\end{abstract}

\section{Introduction}

The vertical propagation of equatorial waves forced in the troposphere plays an important role in the dynamics of the middle atmosphere. While at middle latitudes waves are forced mostly by orography and thermal contrasts, latent heat release in deep convection is mainly responsible for the excitation of waves in the Tropics (Holton 1972; Salby and Garcia 1987; Manzini and Hamilton 1993; Hayashi and Golder 1994; Horinouchi and Yoden 1996; etc.). Convective heating in the tropical troposphere thus represents the main source of energy and momentum in the Tropics: it has a direct influence on the local tropospheric dynamics and a very important indirect effect on the global circulation and the distribution of chemical tracers in the middle atmosphere.

\footnotetext{
* Current affiliation: CIRES, University of Colorado and NOAA/ Climate Diagnostics Center, Boulder, Colorado.

+ The National Center for Atmospheric Research is sponsored by the National Science Foundation.
}

Corresponding author address: Dr. Rolando R. Garcia, NCAR, P.O. Box 3000, Boulder, CO 80307-3000.

E-mail: garcia@ucar.edu
Wave-mean flow interaction is responsible for dynamical phenomena peculiar to the tropical middle atmosphere, like the quasi-biennial oscillation $(\mathrm{QBO})$ of zonal winds, observed in the lower stratosphere (Reed et al. 1961; Naujokat 1986), and the semiannual oscillation (SAO) in the upper stratosphere and mesosphere (Reed 1965; Hirota 1978). Most classic theories of the QBO have attributed the observed accelerations of the mean zonal flow to momentum deposition by large-scale equatorial waves that propagate from the troposphere (Holton and Lindzen 1972; Plumb 1977), although the original Lindzen and Holton (1968) theory relied on small-scale gravity waves.

Mechanistic numerical models developed to simulate the QBO following the classic theories take into account only large-scale waves (zonal wavenumbers less than 5 and periods greater than 5 days). In particular, twodimensional models (Plumb and Bell 1982; Dunkerton 1985; Takahashi 1987; Gray and Pyle 1989) have been successful in simulating the QBO features observed in the meridional plane. A realistic $\mathrm{QBO}$ has also been simulated with a three-dimensional mechanistic model by Takahashi and Boville (1992). However, in order to produce the QBO, Takahashi and Boville had to specify arbitrary-and larger than observed-amplitudes for large-scale Kelvin and Rossby-gravity waves at the tropopause. 

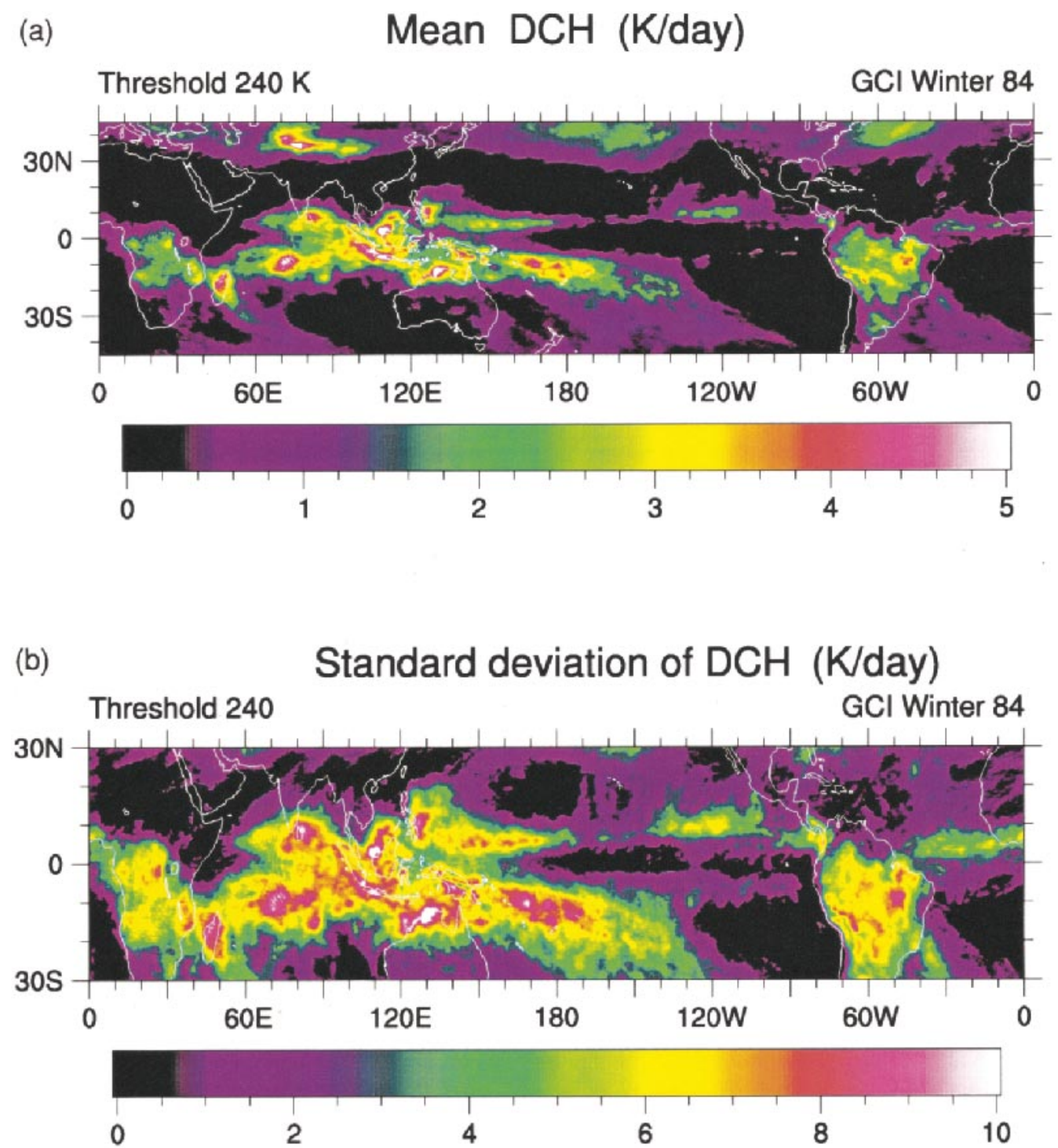

FIG. 1. (a) Time mean DCH in the Tropics and (b) its standard deviation derived from GCI data for the season winter 84 . See text for details. Units are $\mathrm{K} \mathrm{day}^{-1}$.

General circulation models (GCMs) represent a more realistic tool for studying the role of wave-mean flow interaction; they consider a continuous spectrum of waves internally generated in the model and they include all scales resolved by the model grid. However, GCMs have not been successful in the simulation of the QBO despite the fact that large-scale wave amplitudes are reproduced realistically (Hayashi et al. 1984; Boville and Cheng 1988; Boville and Randel 1992). Moreover, although GCMs reproduce the upper-stratospheric SAO, they are not able to simulate a realistic descent of the westerly phase of the oscillation (Sassi et al. 1993).

All of this suggests that the large-scale waves parameterized in mechanistic models and forced in GCMs may not carry sufficient momentum to drive the tropical zonal mean wind oscillations. This hypothesis is supported both by results derived from observations and by modeling. Bergman and Salby (1994) analyzed the heating field derived from observations of tropical convection and inferred that only $50 \%$ of the vertical momentum flux propagating into the stratosphere is carried by largescale waves; the rest is due to higher-frequency, smallerscale gravity waves, with periods shorter than 2 days. Similar results have been obtained by other methods (Sato et al. 1994; Sato and Dunkerton 1997; Tsuda et al. 1994a,b). Dunkerton (1997) reviews some of these observations and argues that the momentum budget of the QBO probably requires contributions from waves over a broad range of wavenumbers and frequencies. A "QBO-like" oscillation was recently obtained by Horinouchi and Yoden (1998) with an "aquaplanet" GCM: they found that, in this model, about $40 \%$ of the mo- 


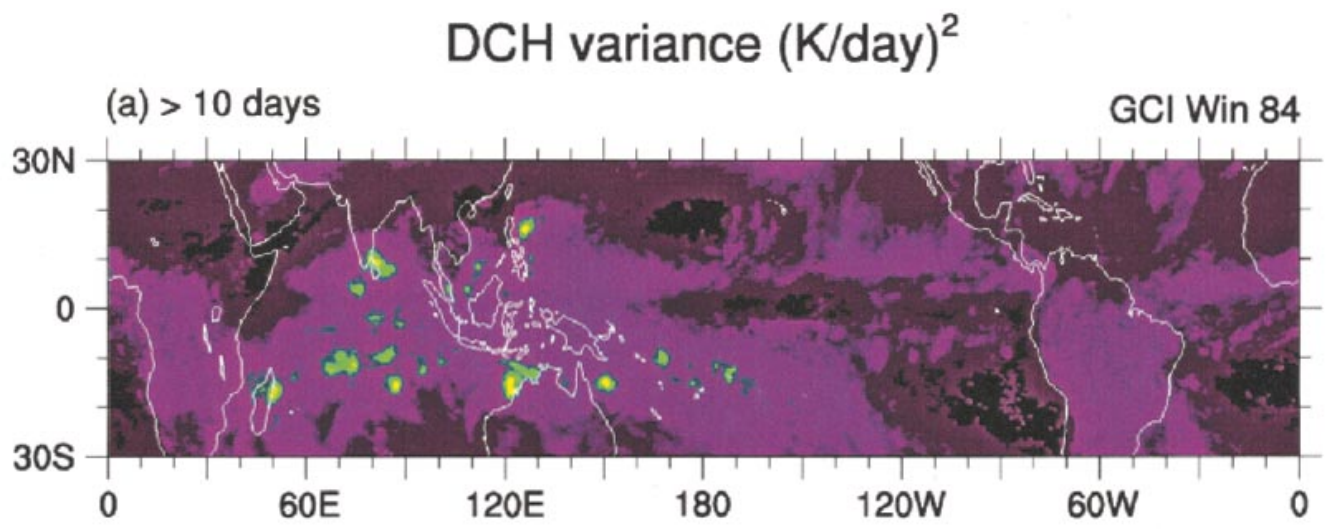

(b) 2 days -10 days

\section{GCI Win 84}

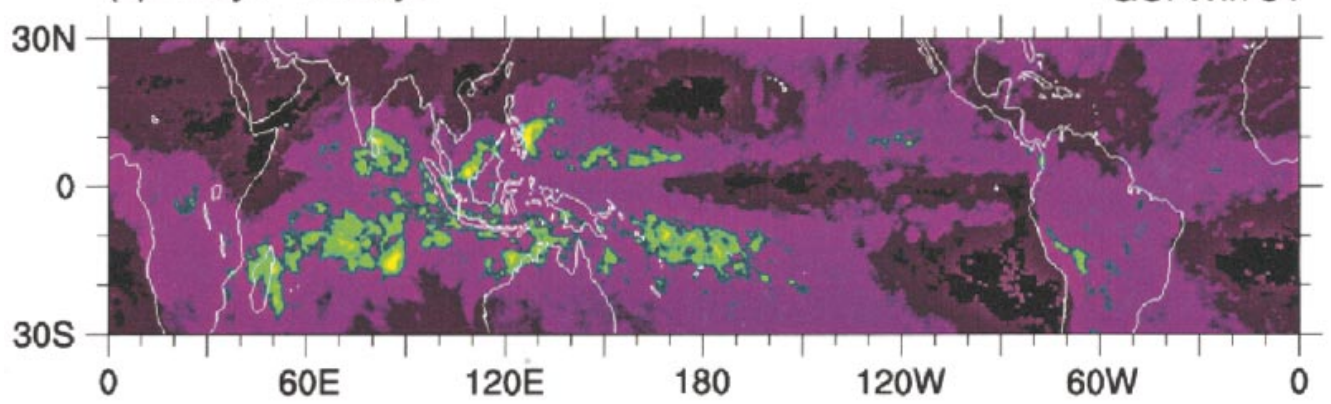

(c) 6 hrs - 2 days

GCI Win 84

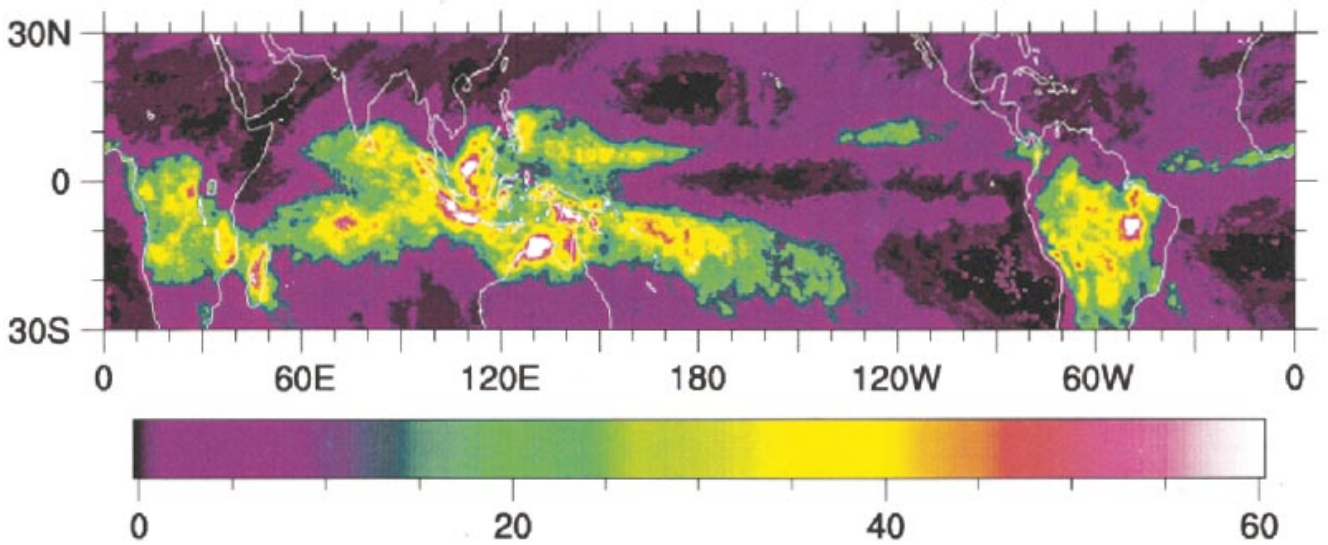

FIG. 2. Variance of the DCH derived from GCI data for winter 84. The variance is shown for three bands of period (a) $T>10$ days, (b) 2 days $<T<10$ days, and (c) $6 \mathrm{~h}<T<2$ days. Units are $\left(\mathrm{K}^{\text {day }}{ }^{-1}\right)^{2}$.

mentum flux carried by vertically propagating waves reaching the lower stratosphere was due to medium- and small-scale gravity waves.

In order to understand the reasons why GCMs have difficulty simulating a realistic $\mathrm{QBO}$ and SAO, and to investigate the momentum budget due to waves on different spatial and temporal scales, we have conducted a detailed analysis of tropical waves forced in the National Center for Atmospheric Research (NCAR) Com- munity Climate Model (CCM3) following the method of Bergman and Salby (1994, hereafter BS94). The convective heating field in CCM3 is decomposed into frequency and zonal wavenumber components and projected onto Hough modes. Then, it is possible to derive the spectrum of waves excited by the heating (Kelvin, Rossby, and gravity waves) and analyze the relative contribution of different waves to the total EliassenPalm (EP) flux. The results of this analysis are then 
compared to the corresponding spectra derived from observations using the method of BS94. The comparison reveals large differences between the observations and CCM3 and may help account for deficiencies in the model's simulation of the middle atmosphere and even the troposphere.

\section{Equatorial wave excitation: Theoretical formulation}

Equatorial waves are oscillations of the atmosphere confined in the Tropics. An equation describing these oscillations can be derived from the linearized primitive equations for eddy motions in a steady, zonally symmetric basic flow, $\bar{u}$, with no latitudinal or vertical shear, and neglecting momentum dissipation:

$$
\begin{aligned}
\bar{D} u^{\prime}-\left(f+\frac{\bar{u} \tan \varphi}{a}\right) v^{\prime}+\frac{1}{a \cos \varphi} \Phi_{\lambda}^{\prime} & =0 \\
\bar{D} v^{\prime}+\left(f+\frac{2 \bar{u} \tan \varphi}{a}\right) u^{\prime}+\frac{1}{a} \Phi_{\varphi}^{\prime} & =0 \\
\frac{1}{a \cos \varphi}\left[u_{\lambda}^{\prime}+\left(v^{\prime} \cos \varphi\right)_{\varphi}\right]+\frac{1}{\rho_{0}}\left(\rho_{0} w^{\prime}\right)_{z}= & 0 \\
\bar{D} \Phi_{z}^{\prime}+w^{\prime} N^{2}= & \frac{J^{\prime}}{H} \kappa \\
& -\alpha \Phi_{z}^{\prime},
\end{aligned}
$$

where $\bar{D}=\partial / \partial t+(\bar{u} / a \cos \varphi) \partial / \partial \lambda, z$ is a log-pressure coordinate, $f=2 \Omega \sin \varphi$ the Coriolis parameter, $H$ the scale height, and $\alpha$ the coefficient for thermal damping represented as Newtonian cooling; $J^{\prime}$ represents the heating rate fluctuations per unit mass and $\kappa=R / c_{p}$. After writing eddy variables in wave form, for example, $u^{\prime}=u_{k}^{\omega}(\varphi, z) e^{i(k \lambda-\omega t)}$, we can combine Eqs. (1a)-(1d) to obtain a single differential equation for the Fourier components of the geopotential, $\Phi_{k}^{\omega}(\mu, z)$ :

$$
\begin{gathered}
\mathcal{C V}\left[\Phi_{k}^{\omega}(\mu, z)\right]-\mathcal{L}_{k}^{\omega}\left[\Phi_{k}^{\omega}(\mu, z)\right] \\
=\frac{i \kappa}{\omega_{D} H} e^{z / H} \frac{\partial}{\partial z}\left[e^{-z / H} J_{k}^{\omega}(\mu, z)\right],
\end{gathered}
$$

where $\mu=\sin \varphi$ and

$$
C=\frac{N^{2}}{a^{2}(2 \Omega)^{2}} .
$$

In the above equation the Doppler-shifted (intrinsic) frequency, for $\bar{u}=U \cos \varphi$, is given by

$$
\omega_{D}=\omega-\frac{k}{a} U .
$$

For an atmosphere with constant lapse rate, the differential operators in Eq. (2) acting on an arbitrary function $F$ are

$$
\begin{aligned}
\mathcal{V}[F]= & e^{z / H} \frac{\partial}{\partial z}\left[e^{-z / H}(\Gamma) \frac{\partial F}{\partial z}\right] \text { and } \\
\mathcal{L}_{k}^{\omega}[F]= & \frac{\partial}{\partial \mu}\left[\frac{1-\mu^{2}}{\sigma^{2}-\mu^{2}} \frac{\partial F}{\partial \mu}\right] \\
& +\frac{1}{\sigma^{2}-\mu^{2}}\left[\left(\frac{k}{\sigma}\right) \frac{\sigma^{2}+\mu^{2}}{\sigma^{2}-\mu^{2}}-\frac{k^{2}}{1-\mu^{2}}\right] F,
\end{aligned}
$$

where $\sigma=\omega_{D} / 2 \Omega$ and the thermal damping is included in the parameter

$$
\Gamma=1+\frac{i \alpha}{\omega_{D}} .
$$

The operator $\mathcal{L}_{k}^{\omega}$ defines Laplace's tidal equation:

$$
\mathcal{L}_{k}^{\omega}\left[\Theta_{k n}^{\omega}(\mu)\right]+\varepsilon_{k n}^{\omega} \Theta_{k n}^{\omega}(\mu)=0,
$$

where $n$ is a meridional wavenumber. Solutions to (8) form a complete set of orthogonal functions $\left\{\Theta_{k n}^{\omega}(\mu)\right\}_{n}$, the Hough modes, with eigenvalues $\varepsilon_{k n}^{\omega}$; the Hough functions represent the normal modes of oscillation of a shallow, rotating atmosphere. The solutions are thoroughly described in Longuet-Higgins (1968); they consist of three manifolds, describing eastward-propagating gravity waves (including Kelvin waves), westwardpropagating gravity waves, and Rossby waves. Eastward-propagating Rossby-gravity waves are included under the eastward gravity wave manifold, while westward-propagating Rossby-gravity waves are included in the Rossby manifold. For each manifold two asymptotic regimes exist: in the limit of $\varepsilon \rightarrow \infty$ the solutions are trapped near the equator and they can be expressed as a linear combination of Hermite functions; for $\varepsilon \rightarrow$ 0 they move toward the Poles and are easily expressed as linear combinations of associated Legendre functions. The complete set of Hough modes includes solutions of Eq. (8) for negative eigenvalues $\varepsilon_{k n}^{\omega}$; however, these modes are confined near the Poles and we neglect them as they are not efficiently excited by tropical forcing.

If mechanical friction is neglected, Eq. (2) is separable and can be solved by projecting the Fourier components of geopotential and heating term onto Hough modes:

$$
\begin{aligned}
\Phi_{k}^{\omega}(\mu, z) & =\sum_{n} \Phi_{k n}^{\omega}(z) \Theta_{k n}^{\omega}(\mu) \\
J_{k}^{\omega}(\mu, z) & =\sum_{n} Q_{k n}^{\omega}(z) \Theta_{k n}^{\omega}(\mu),
\end{aligned}
$$

where the Hough modes are normalized such that $\int_{-1}^{1}\left|\Theta_{k n}^{\omega}(\mu)\right|^{2} d \mu=1$.

The resulting equations for the vertical structure of the geopotential, including boundary conditions, are

$$
\begin{gathered}
\frac{\partial^{2}}{\partial z^{2}} \hat{\Phi}_{k n}^{\omega}+\left(m_{k n}^{\omega}\right)^{2} \hat{\Phi}_{k n}^{\omega}=\hat{Q}_{k n}^{\omega} \\
\frac{\partial}{\partial z} \hat{\Phi}_{k n}^{\omega}+\left(\frac{1}{2 H}-\frac{N^{2}}{g \Gamma}\right) \hat{\Phi}_{k n}^{\omega}=0 \quad(z=0) \\
\text { finite energy } \\
(z \rightarrow \infty),
\end{gathered}
$$


where $\hat{\Phi}_{k n}^{\omega}(z)$ and $\hat{Q}_{k n}^{\omega}(z)$ are the following auxiliary variables introduced to cast the vertical structure equation in standard form:

$$
\begin{aligned}
& \hat{\Phi}_{k n}^{\omega}(z)=e^{-z / 2 H} \Phi_{k n}^{\omega}(z) \\
& \hat{Q}_{k n}^{\omega}(z)=e^{z / 2 H} \frac{i \kappa}{\omega_{D} \Gamma H} \frac{\partial}{\partial z}\left[e^{-z / H} Q_{k n}^{\omega}(z)\right] .
\end{aligned}
$$

The vertical wavenumber for the component $\Phi_{k n}^{\omega}$ is

$$
m_{k n}^{\omega}=\operatorname{sgn}(\omega)\left(\frac{\varepsilon_{k n}^{\omega} C}{\Gamma}-\frac{1}{4 H^{2}}\right)^{1 / 2} .
$$

The solution to the problem (11) can be found using the Green's functions corresponding to the differential operator in (11a), with the same method presented in Salby and Garcia (1987) for a frictionless isothermal atmosphere. In particular, an analytical solution exists if we assume the heating to be separable, with a vertical profile $q(z)$ and horizontal structure defined by Hough components $Q_{k n}^{\omega}$ :

$$
Q_{k n}^{\omega}(z)=Q_{k n}^{\omega} q(z) .
$$

This assumption corresponds to a vertically coherent heating field and, as we will see later, it is reasonable for our analysis. The complete solution for the geopotential in the near field above the heating can then be expressed as

$$
\Phi_{k n}^{\omega}(z)=i \frac{\kappa Q_{k n}^{\omega}}{\omega_{D} \Gamma H} P_{m}^{-} e^{(1 / 2 H-i m) z},
$$

where $P_{m}^{-}$is the projection of the heating onto to the vertical structure of the waves. The far field dynamical response to heating is subject to the influence of wave absorption and refraction, and is described in Garcia and Salby (1987).

In general, the vertical projection $P_{m}^{-}$depends on the vertical profile of the heating. Salby and Garcia (1987) found that, for a sufficiently smooth vertical heating profile, the projection $P_{m}^{-}$is more sensitive to the heating depth than to the detailed shape of the profile. The vertical projection for the geopotential is a function with multiple maxima: the response is dominated by vertical wavelengths corresponding approximately to twice and two-thirds the heating depth (the first and second projection responses, respectively; see Fig. 2 of BS94). The vertical projection $P_{m}^{-}$for a heating profile proportional to $\exp \left(i_{0} z\right)$ in the region $\left(z_{0}, z_{0}+\Delta z\right)$, with characteristic wavenumber $m_{0}$, is given by

$$
\begin{aligned}
\mathcal{P}\left(m_{0}\right)_{m}^{-}=\frac{\Delta z}{2 m} e^{i m[z 0+(\Delta z / 2)]} & \left(m-\frac{i}{2 H}\right) \operatorname{sinc}\left[\frac{(m+\hat{m})}{\Delta m}\right] e^{i m\left[z_{0}+(\Delta z / 2)\right]} \\
& \left.-\frac{\left[m-i\left(\frac{1}{2 H}-\frac{N^{2}}{g \Gamma}\right)\right]}{\left[m+i\left(\frac{1}{2 H}-\frac{N^{2}}{g \Gamma}\right)\right]}\left(m+\frac{i}{2 H}\right) \operatorname{sinc}\left[\frac{(m-\hat{m})}{\Delta m}\right] e^{-i m\left[z_{0}+(\Delta z / 2)\right]}\right\},
\end{aligned}
$$

where

$$
\hat{m}=m_{0}+\frac{i}{2 H}
$$

and $\operatorname{sinc} x=(\sin \pi x) / \pi x$.

The dynamical near-field response is completely described by the geopotential function: Fourier components of both wind and temperature can be easily derived from the geopotential components. The Fourier components of the vertical EP flux can be calculated from

$$
\left\{F_{z}(\varphi)\right\}_{k}^{\omega}=\left[f \frac{R}{H} \frac{v_{k}^{\omega} T_{k}^{\omega}}{N^{2}}-u_{k}^{\omega} w_{k}^{\omega}\right] .
$$

Note that (18) differs by a factor of $\rho_{0} a$ from the conventional definition of $F_{z}$ (e.g., Andrews et al. 1987); however, (18) is adopted here because it facilitates comparison with other, recent estimates of $F_{z}$ that are discussed later on.

In the near field above the source region, an approximate expression for the Hough components $\left\{F_{z}\right\}_{k n}^{\omega}$ can be found by neglecting thermal dissipation:

$$
\begin{aligned}
\left\{F_{z}\right\}_{k n}^{\omega} & =\frac{1}{N^{2}} \operatorname{Re}\left\{\frac{\partial \Phi_{k n}^{\omega}}{\partial \lambda}\left(\frac{\partial \Phi_{k n}^{\omega}}{\partial z}\right)^{*}\right\} \\
& =-\frac{1}{a N^{2}} k \operatorname{Re}\{m\}\left|\Phi_{k n}^{\omega}(z)\right|^{2} .
\end{aligned}
$$


(a)

Mean tropospheric heating rate (K/day)

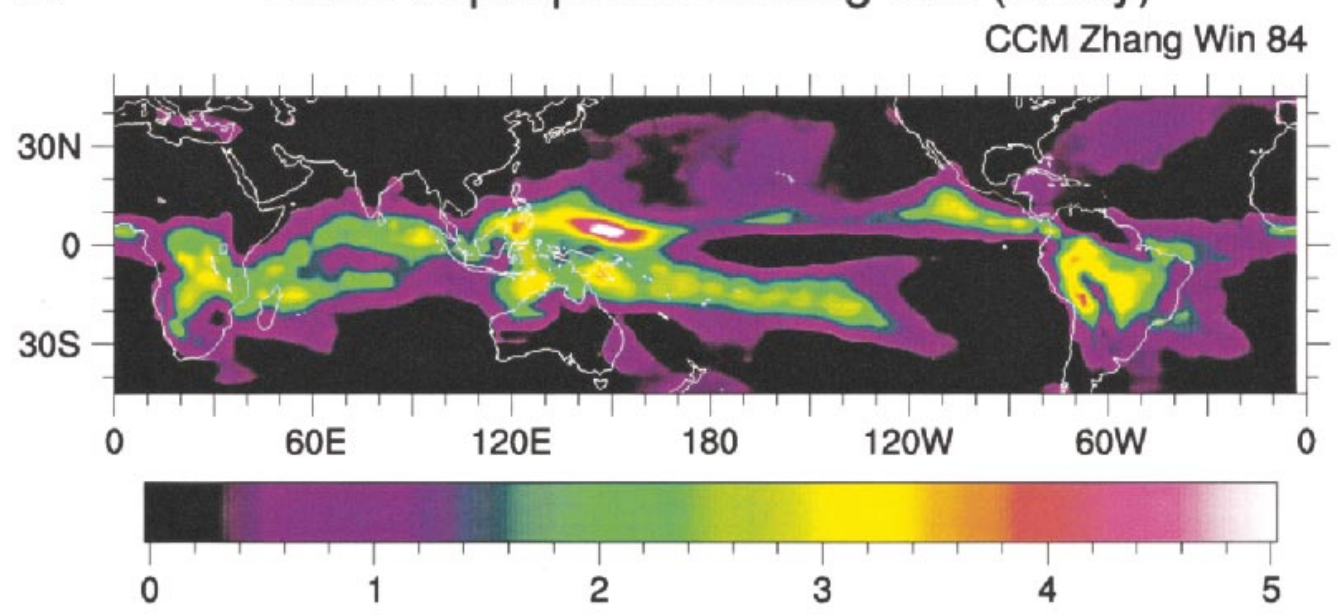

(b) Standard deviation of heating rate (K/day)

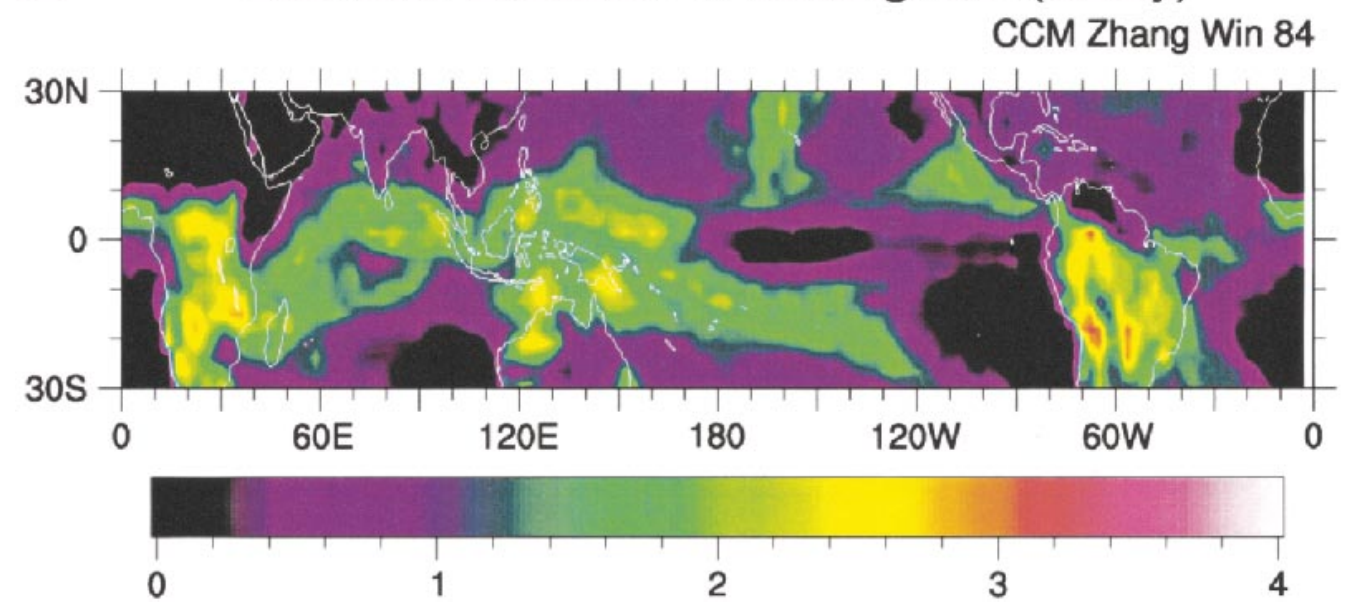

FIG. 3. As in Fig. 1, but for the heating rate calculated by CCM3 with the Zhang-McFarlane convective scheme.

Here, $F_{z}$ represents the pseudomomentum flux due to vertically propagating waves originating in the upper troposphere; it is a crucial dynamical variable for studying the interaction between waves and the mean flow in the middle atmosphere. Equation (19) follows in straightforward fashion from (1) and the definition (18) if dissipation of momentum and temperature are neglected. Although $F_{z}$ can be obtained directly and without approximations from (18), Eq. (19) has the advantage that it allows $F_{z}$ to be broken down into the contributions of individual Hough components, $\Phi_{k n}^{\omega}$ (see Horinouchi and Yoden 1998).

\section{Deep convection in the Tropics: Observations and modeling}

An efficient mechanism for wave excitation in the Tropics is the release of latent heat in deep convection.
In order to understand possible deficiencies in modeling wave excitation in the Tropics, a preliminary analysis of this wave source has been conducted, comparing the frequency distribution of the tropical heating field in CCM3 with observations.

\section{a. Observations of deep convection}

One strategy for studying deep convection could be to analyze observed precipitation data (e.g., Gray and Jacobson 1977), which is directly related to the total heat released in the tropospheric column above the precipitation region. However, observations of precipitation are not widely and uniformly distributed in the Tropics and they do not permit a detailed study on a large spatial scale. Indirect estimates of precipitation can 


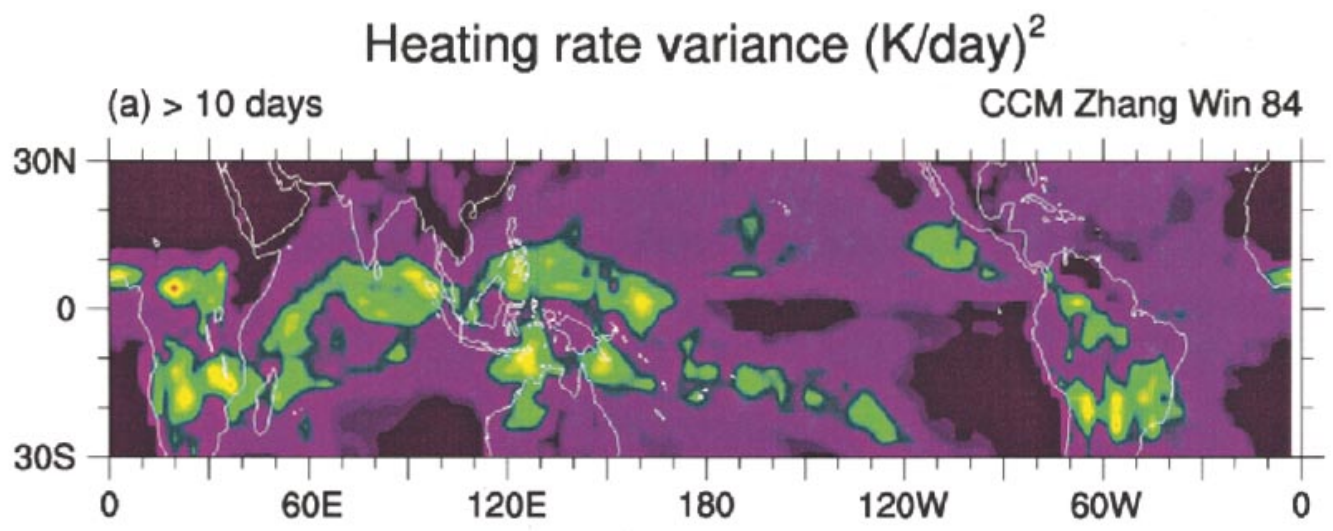

(b) 2 days -10 days

\section{CCM Zhang Win 84}

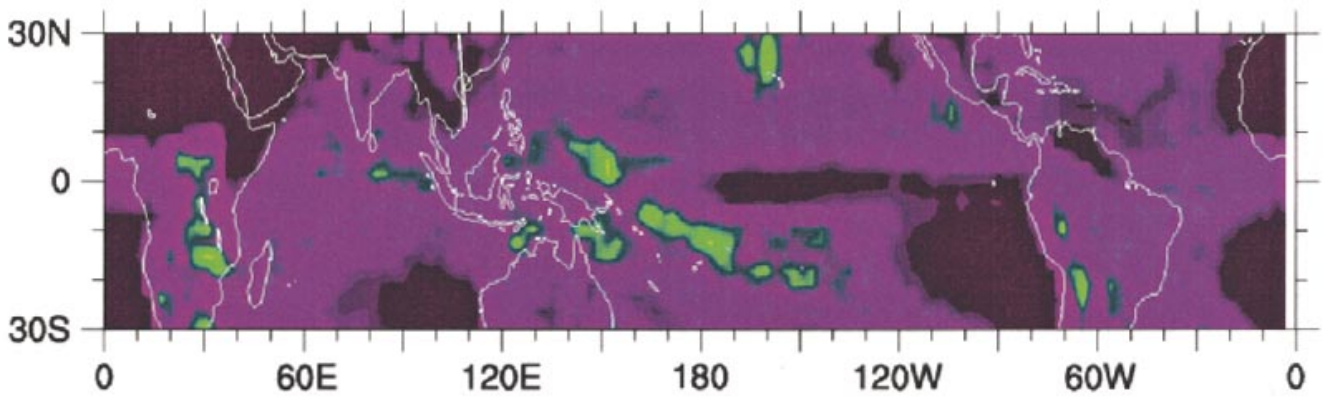

(c) 6 hrs - 2 days

CCM Zhang Win 84

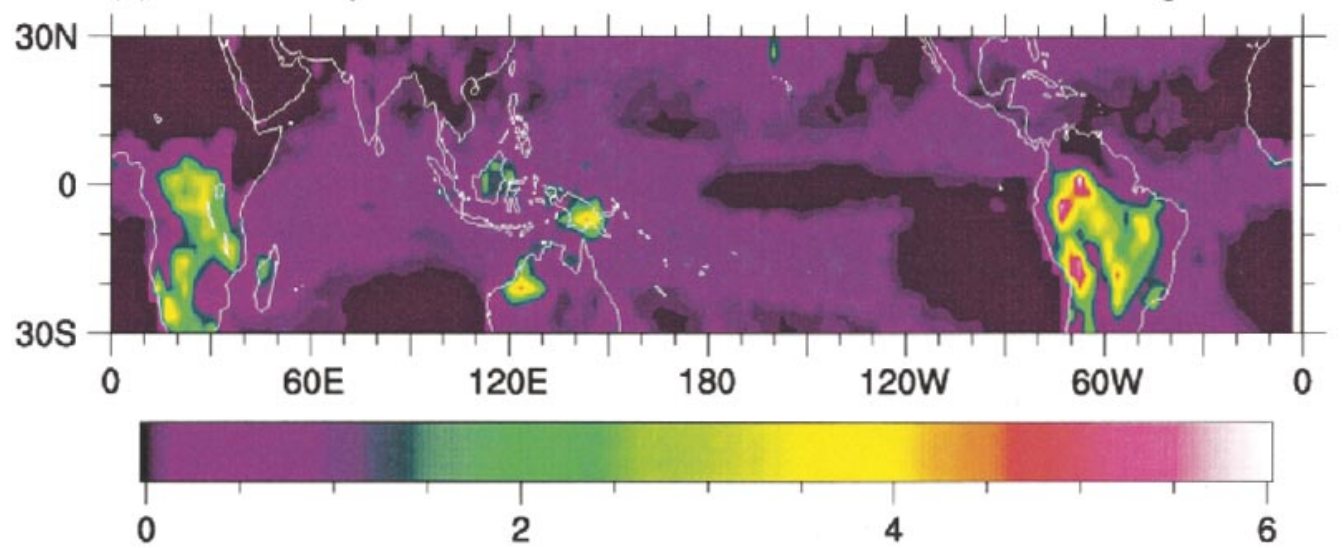

FIG. 4. As in Fig. 2, but for the variance of the heating rate calculated by CCM3 with the Zhang-McFarlane convective scheme.

be obtained from satellite observations of visible and IR radiation emitted by clouds (see, e.g., Arkin and Ardanuy 1989). In the Tropics, where precipitation is strongly linked to deep convection, observations of outgoing longwave radiation (OLR) are a useful proxy for convective activity (Heddinghaus and Krueger 1981; Liebmann and Hartmann 1982; Rossow 1989). Because deep convection is associated with high, cold clouds, indices of convective precipitation can be based upon the presence of cloud cover at temperatures below some suitably defined threshold. Furthermore, the correlation between tropical rainfall rates derived in this manner and ground-based observations of precipitation is quite good over a wide range of spatial and temporal scales (Richards and Arkin 1981; Elbert et al. 1996).

A complete set of data with high spatial and temporal 
resolution can be obtained only by collecting simultaneous observations from different satellites. With this purpose in mind, the International Satellite Cloud Climatology Project (ISCCP) (Schiffer and Rossow 1983) was developed. ISCCP data have been used by Salby and coworkers to create a synoptic dataset of the global cloud field, the global cloud imagery (GCI) (Tanaka et al. 1991; Salby et al. 1991). The observations refer to $11-\mu \mathrm{m}$ IR radiance, which is then converted into equivalent blackbody brightness temperature. The dataset provides high-resolution global images of radiances observed every $3 \mathrm{~h}$; each image is composed of $(512)^{2}$ pixels, giving a resolution of $0.35^{\circ}$ in latitude and $0.7^{\circ}$ in longitude. The mean brightness temperature (TBR) obtained from the radiances is a measure of the temperature at the top of the clouds. A detailed analysis of the TBR observed for the fall season 1983 is presented in Salby et al. (1991).

Our analysis focuses on the role of tropical heating in wave excitation and requires discrimination of deep convective clouds from the total set of observations of IR radiance. For this purpose, a deep convective activity (DCA) index can be defined (Hendon and Woodberry 1993):

$$
\mathrm{DCA}= \begin{cases}240 \mathrm{~K}-\mathrm{TBR} & \text { if } \mathrm{TBR}<240 \mathrm{~K} \\ 0 & \text { otherwise. }\end{cases}
$$

The choice of threshold temperature is based on a comparison between observed precipitation and TBR; Richards and Arkin (1981) estimated that observations of TBR $<240 \mathrm{~K}$ give a good correlation between convective cloud coverage and precipitation. The above definition has been used by BS94 and differs only slightly from that of Hendon and Woodberry, who chose $230 \mathrm{~K}$ as the threshold temperature.

The DCA index can be related to precipitation and consequently to the heat released during condensation of water in the convective column. For the purpose of comparing the convective heating field from CCM3 with observations, we have converted the variable DCA to a deep convective heating rate (DCH). The DCH has units of Kelvins per day; the details of the conversion are described in the appendix. The time series of the DCH analyzed covers the period 14 November 1983 20 March 1984, referred to as "winter 84" in what follows. This period was chosen because it coincides with that analyzed in BS94, whose results can serve as a check on our analysis technique, which differs slightly from Bergman and Salby's.

Figure 1 shows the time mean and the standard deviation of $\mathrm{DCH}$ in the tropical region. The intertropical convergence zone (ITCZ) appears clearly and other convective structures are seen over tropical South America and Africa, Madagascar, Indonesia, and northern Australia. These structures are more sharply localized in space than the ones resulting from the analysis of TBR (Salby et al. 1991, their Fig. 5). High values of mean
DCH in the Himalayan region do not represent deep convection, as borne out by the fact that they are not associated with significant variability in time; instead, they are related to cold surface temperatures in that region at this time of the year. The standard deviation of $\mathrm{DCH}$ about the time mean for winter 84, shown in Fig. 1b, illustrates the high variability of convective activity in the Tropics. In order to establish the nature of this variability, we analyze the contribution to total variance for three different frequency bands: low frequency (periods $T>10$ days), medium frequency $(2<$ $T<10$ days), and high frequency ( $6 \mathrm{~h}<T<2$ days). The DCH field was decomposed into its Fourier components, with Nyquist frequency of 4 cycles day ${ }^{-1}$ (cpd), after removing a linear trend and applying a Hanning window (10\% cosine taper). Figure 2 shows clearly that the variance of $\mathrm{DCH}$ is mainly due to high-frequency components, unlike the variance of TBR, which shows an important contribution from both low and high frequencies (cf. Salby et al. 1991, their Fig. 6). This feature of the DCH is very important because it shows that, despite the fact that the time mean is organized on large and medium scales, the deep convection is not at all a steady process and thus is able to excite high-frequency waves that can propagate efficiently into the middle atmosphere.

\section{b. The CCM3}

The Community Climate Model (CCM) is a general circulation model developed at the National Center for Atmospheric Research for global dynamics and climate studies. The current version, CCM3, is thoroughly described by Kiehl et al. (1996). CCM3 is a spectral threedimensional model with T42 horizontal truncation; it uses 18 vertical levels extending from the surface up to about $3 \mathrm{mb}$.

In order to study the excitation of equatorial waves in the model, we analyzed the model variable corresponding to deep convective heating. This variable, ZMDT, represents the temperature tendency due to net latent heat released during condensation of air masses in deep convection, as parameterized by Zhang and McFarlane (1995):

$$
\begin{aligned}
\mathrm{ZMDT} & =\left(\frac{\partial T}{\partial t}\right)_{\text {conv. }} \\
& =\frac{1}{c_{p}}\left[L_{c}(C-E)-\frac{1}{\rho_{0}} \frac{\partial}{\partial z} M_{c}\left(S_{c}-S\right)\right],
\end{aligned}
$$

where $L_{c}$ is the latent heat of condensation for water vapor, and $C$ and $E$ are, respectively, the condensation and evaporation rates. The second term on the right represents the divergence of static energy mass flux, where $M_{c}$ is the net convective mass flux and $S_{c}, S$ are, respectively, the mean cloud and large-scale dry static energy. Instead of analyzing the full three-dimensional 
field of ZMDT, we characterize its horizontal structure by the vertical average of ZMDT over the depth of the troposphere:

$$
\langle\mathrm{ZMDT}\rangle=\frac{1}{c_{p}} \frac{1}{\Delta z} \int_{0}^{z_{t}} L_{c}(C-E) d z,
$$

where $z_{t}$ is the altitude of the tropopause. We then assume a vertical profile with an effective depth of approximately $10 \mathrm{~km}$ :

$$
q(z)= \begin{cases}1 & \text { for } 0.7<z<11.0 \mathrm{~km} \\ 0 & \text { elsewhere }\end{cases}
$$

which is equivalent to setting $m_{0}=0$ in the solution for the vertical projection of the heating, Eq. (16). As noted above, if the heating field has a smooth vertical profile, the dynamical response to the forcing is insensitive to the detailed shape of the profile. Analysis of ZMDT (21) in the Tropics showed that the vertical distributions of both the mean and the standard deviation of ZMDT are rather uniform, smoothly varying with altitude, and extend over a depth slightly larger than 10 $\mathrm{km}$. Therefore, the choice of $\langle\mathrm{ZMDT}\rangle$ (22) and the vertical profile (23) for our analysis is reasonable. It also makes possible a direct comparison between $\langle$ ZMDT $\rangle$ and the heating field, DCH, inferred from the GCI observations (whose vertical profile is not generally known): we simply assume that the same vertical profile (23) applies to both model results and observations.

Another variable connected to convection is the brightness temperature of clouds, TBR, which can be calculated from the OLR field. The same procedure used to convert observed TBR into a DCA index and thence to DCH could have been applied to the OLR field produced by CCM3. This would have the advantage of yielding a two-dimensional field analogous to the DCH obtained from cloud observations. However, the correlation between OLR and precipitation in the model turns out to be very weak, in contrast to the behavior of the real atmosphere, where cold, high clouds (TBR $<240 \mathrm{~K})$ are associated statistically with deep convection and heavy precipitation. ${ }^{1}$ Since we are interested in studying the forcing of waves by latent heat release, it is clear that the OLR field generated by the model (and any quantity derived from it) is not suitable for our analysis. On the other hand, $\langle$ ZMDT $\rangle$ is a direct measure of convective forcing in CCM3.

\footnotetext{
${ }^{1}$ The reason why OLR and precipitation are poorly correlated in CCM3 is not clear. One possibility is that in CCM3 convective cloudiness is defined according to a random-overlap model; i.e., clouds are assumed to overlap randomly to give the total fraction of a grid box that is cloud covered. This produces higher effective OLR than would be expected from real convective clouds because the "cloudtop" radiation comes from different levels in the grid box rather than from a single-level high in the troposphere. Thus, the strong relationship between very cold convective clouds and high precipitation rates breaks down.
}

\section{c. Convective heating in CCM3}

Figure 3 shows the time mean and standard deviation of the convective heating field for winter 84 obtained with CCM3, wherein deep convection is parameterized by means of the Zhang-McFarlane scheme mentioned above. The scheme of Hack (1994), which was used in an earlier version of the Community Climate Model (CCM2), is also present in CCM3; however, its role in CCM3 is only to remove any residual convective instability left after application of the Zhang-McFarlane scheme, and to handle shallow convection. We shall refer to the standard version of CCM3 as "CCM-Z" in what follows. In the context of the simulation, winter 84 means that the model was run with sea surface temperatures (SSTs) taken from observations for this period. Insofar as the SST distribution influences the intensity and location of tropical convection, the winter 84 simulation should be comparable to observations of deep convection for the same period.

The spatial distribution of $\langle\mathrm{ZMDT}\rangle$ for CCM-Z is generally in good agreement with the time mean DCH in Fig. 1, although there are some differences in the Indonesian and western Pacific regions. The convective heating $\langle Z M D T\rangle$ also shows variability about the mean value; however, the variability is considerably smaller than in the observations, is mainly localized over the continents, and is scarcely present over oceanic regions, unlike the convective heating, $\mathrm{DCH}$, derived from observations. As a consequence, the standard deviation of $\langle$ ZMDT $\rangle$, averaged over the Tropics $\left(21^{\circ} \mathrm{S}-21^{\circ} \mathrm{N}\right)$, is much smaller than the standard deviation derived from observations, while the mean values for modeled and observed tropical heating are approximately the same. Figure 4 shows the breakdown of power into three frequency bands. Power is strongly concentrated at low frequencies, except for regions over the tropical continents, where variance associated with the diurnal cycle is large. (Note, by the way, that the variance scale in Fig. 4 is 10 times smaller than for the GCI data in Fig. 2.)

The comparison between observed and modeled convective heating illustrates the underestimation of highfrequency variability in CCM3 using the Zhang-McFarlane convection scheme, despite the fact that the mean values of convective heating are well represented. This could result in unrealistically weak excitation of equatorial waves in the model and points to the need to investigate in detail the spectrum of waves excited by tropical deep convection.

To assess the sensitivity of $\mathrm{CCM} 3$ convective heating to the parameterization scheme employed, we analyzed the output of a second simulation covering the winter 84 season. In this simulation, which we call "CCM-H," the Zhang-McFarlane scheme is completely removed and the Hack (1994) scheme becomes the only convective parameterization, handling both shallow and deep convection. This makes CCM-H more similar to CCM2 

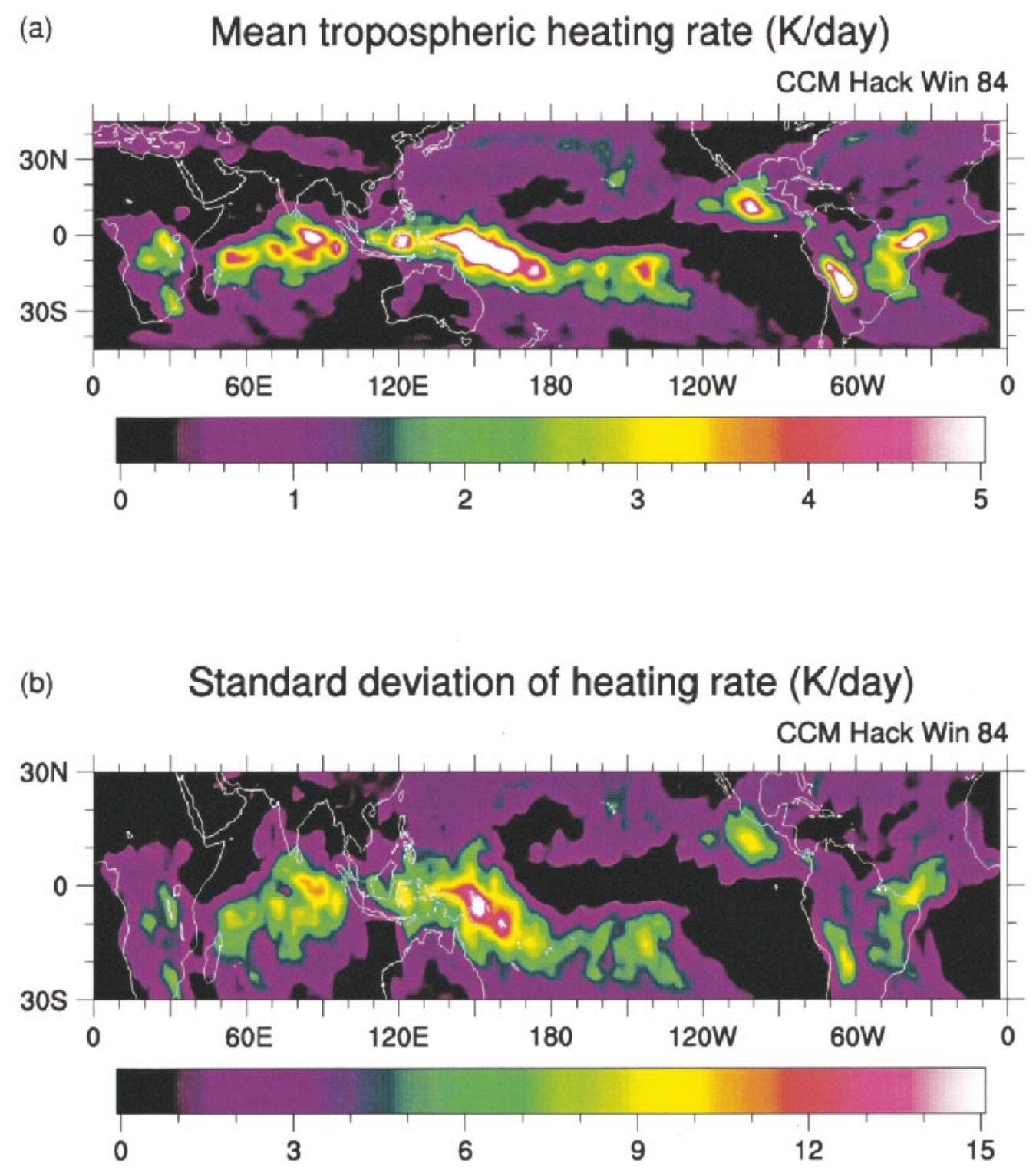

FIG. 5. As in Fig. 1, but for the heating rate calculated by CCM3 with the Hack convective scheme.

than to the standard version of $\mathrm{CCM} 3$, at least in the Tropics.

Figure 5 shows the mean deep convective heating field $\langle$ ZMDT $\rangle$ and its standard deviation for winter 84 as simulated by CCM-H. The spatial structure of the mean field is similar to that observed (Fig. 1) and also to the mean convective heating field obtained from $\mathrm{CCM}-\mathrm{Z}$, except for the unrealistically strong convection in the western Pacific near $150^{\circ} \mathrm{E}$. In addition, the standard deviation of $\langle$ ZMDT $\rangle$ is much larger than in the Zhang scheme (Fig. 3). However, variability in CCM-H is more strongly concentrated over the oceans than in $\mathrm{CCM}-\mathrm{Z}$ or in the GCI data, wherein regions of very strong variability are present over the tropical continents.

A more detailed breakdown of the distribution of heating variance in the CCM-H simulation is presented in Fig. 6 (where the variance scale is the same as in Fig. 2, and larger than in Fig. 4). In CCM-H, the variability of the heating field is almost completely confined to low and medium frequencies. Figure $6 \mathrm{c}$ shows that the diurnal contribution to the variability is negligible with respect to the contribution of lower frequencies. The same conclusion can be reached by noting the lack of variability over the continents for the standard deviation of the heating field (Fig. 5b).

Table 1 summarizes the time mean and variance statistics of the heating field obtained from the GCI data and the two CCM3 simulations. There is a close correspondence among the three time mean values; the same is not true, however, of the total variance or its distribution as a function of period. The total variance 


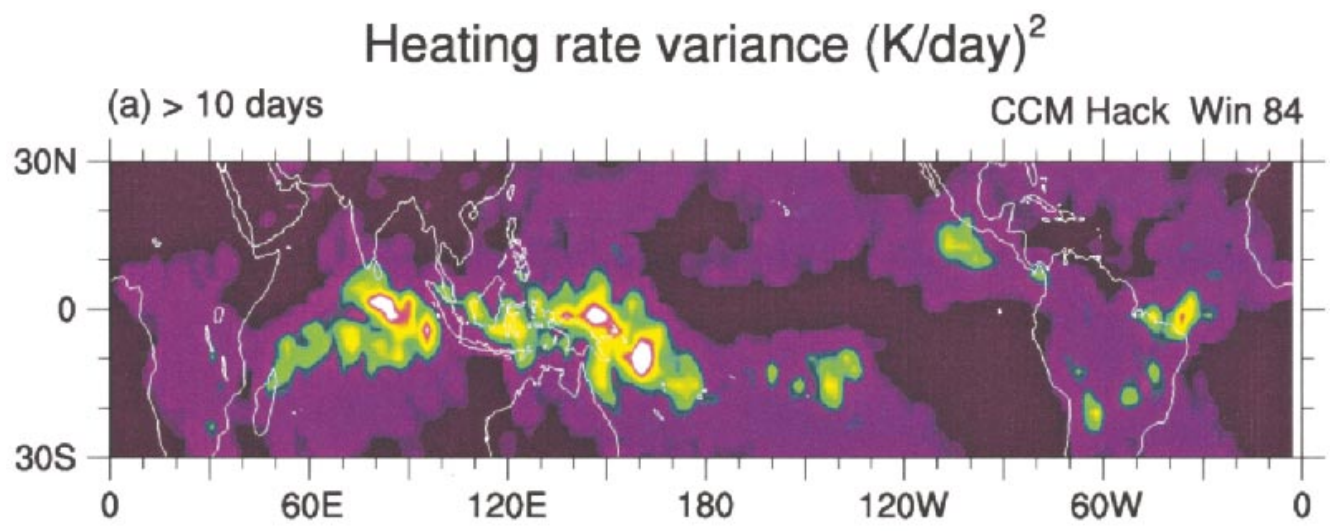

(b) 2 days - 10 days

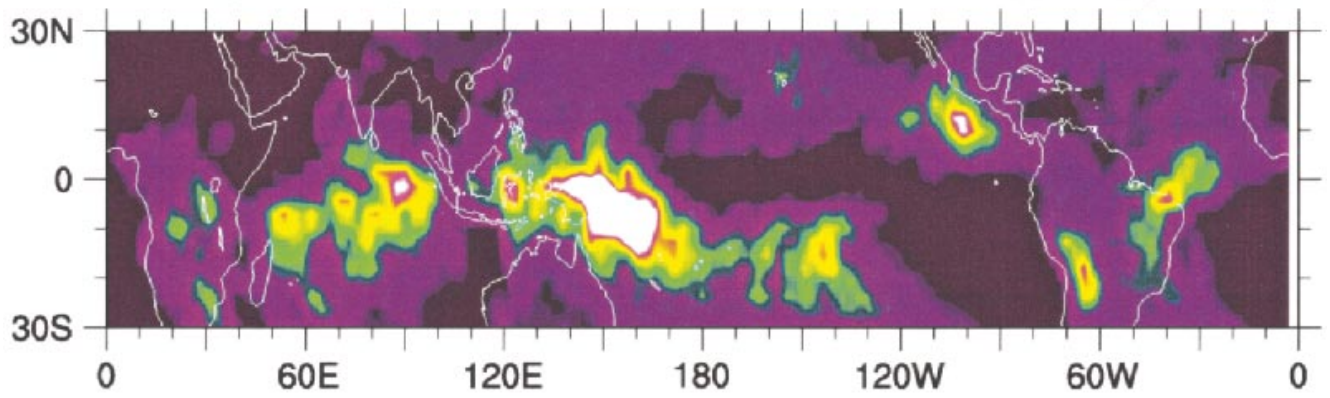

(c) 6 hrs - 2 days

CCM Hack Win 84

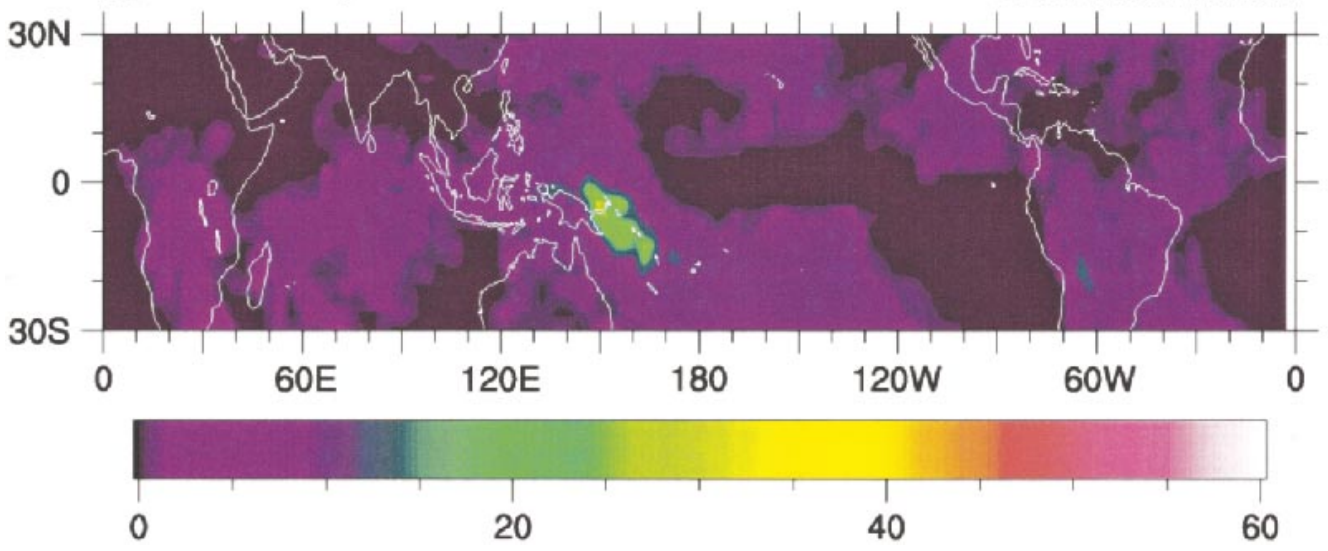

FIG. 6. As in Fig. 2, but for the variance of the heating rate calculated by CCM3 with the Hack convective scheme.

of convective heating in CCM-Z is much less than that inferred from GCI data, as already noted in connection with Fig. 3. Total heating variance in CCM-H, on the other hand, is comparable to that inferred from the GCI, but its distribution with frequency is quite different: $\mathrm{CCM}-\mathrm{H}$ variance is concentrated at periods longer than 2 days (where it even exceeds that in the GCI) but is much smaller at shorter periods (where it is less than $15 \%$ of the variance in the GCI).
The comparison of the spatial distribution of variance between $\mathrm{DCH}$ and the heating field obtained from CCM3 also indicates that convection in CCM3 is organized on a larger spatial scale than in the observations. An analysis of the power spectrum of the heating field as a function of the zonal wavenumber showed that this is only partially an artifact of model resolution. GCI data show a very broad distribution of heating power down to small scales $(k>60)$, with only $40 \%$ of the 
TABLE 1. Mean convective heating and variance averaged over the tropical region $21^{\circ} \mathrm{N}-21^{\circ} \mathrm{S}$, as derived from observations (GCI) and the CCM3 model, with both the Zhang-McFarlane (CCM-Z) and Hack (CCM-H) convective schemes.

\begin{tabular}{lrcc}
\hline \hline & GCI & CCM-Z & CCM-H \\
\hline Mean $\left(\mathrm{K} \mathrm{day}^{-1}\right)$ & 1.0 & 1.1 & 1.1 \\
Total variance $\left(\mathrm{K} \mathrm{day}^{-1}\right)^{2}$ & 17.6 & 1.5 & 15.3 \\
Variance: $\left(\mathrm{K} \mathrm{day}^{-1}\right)^{2}$ & & & \\
$T>10$ days & 2.2 & 0.7 & 5.5 \\
$2<T<10$ days & 4.4 & 0.4 & 8.3 \\
$6 \mathrm{~h}<T<2$ days & 11.0 & 0.4 & 1.5 \\
\hline
\end{tabular}

variance carried by components with zonal wavenumber $k<15$. Although in CCM3 heating power is limited to $k \leq 64,{ }^{2}$ the spatial variability of the heating is sensitive to the convective scheme adopted: in CCM-Z the heating variability is due mostly to large- and medium-scale components $(k<15)$, while in CCM-H these components contribute only about $50 \%$ of the total variance.

The foregoing results illustrate the sensitivity of the calculated convective heating field to the parameterization scheme adopted in the model. They also emphasize the need for a more thorough analysis of the modeled heating field in order to compare spectra of convectively forced equatorial waves with those derived from observations.

\section{Wave forcing by deep convection}

A detailed analysis of the equatorial wave source in CCM3 can be performed by decomposing the convective heating field into Hough modes. The near-field dynamical response of the atmosphere to the forcing can then be evaluated in terms of geopotential and EP flux components, following the formulation of BS94, as described in section 2. The results from CCM3 simulations with the Zhang-McFarlane and the Hack convective schemes are analyzed in this manner and then compared to the wave spectra obtained from the GCI observations.

As explained earlier, we have adopted a single heating profile, Eq. (23), for both the model simulations and the GCI data, since the vertical profile of the latter is not known. Thus, model heating is represented by the vertical integral of the convective heating rate, Eq. (22), times the profile $q(z)$ specified by Eq. (23), and observed heating by the variable DCH [Eq. (A4) of appendix] times the same vertical profile. This facilitates comparison of model results and observations, although it ignores any effects due to differences in the profile of the heating among the model simulations and the observations. In all of our calculations, we have assumed

\footnotetext{
${ }^{2}$ Convective heating in CCM3 is computed on a spatial grid with 128 nodes in the zonal direction. Thus, it is possible to obtain spectra of the heating with $k=64$ resolution. However, the model dynamics are represented in spectral form, with T42 resolution, so only the firs 42 zonal harmonics of the heating affect the dynamical calculations.
}

constant $N^{2}=1.2 \times 10^{-4} \mathrm{~s}^{-2}$ and thermal damping rate $\alpha=0.1$ day $^{-1}$. We also neglected the background zonal wind $U$. The effects of these assumptions are discussed in BS94.

Preliminary operations on the time series to be analyzed are the removal of any linear trend and application of meridional windowing to the heating field in order to discriminate deep convective clouds in the Tropics from cold surface temperatures at higher latitudes, using the function $W(\varphi)=e^{-\left(\varphi / \varphi_{0}\right)^{4}}$ with $\varphi_{0}=25^{\circ}$. A $10 \%$ cosine taper was also applied to the time series (GCI and CCM3) composed of 1024 samples each (128 days $\times 8$ samples per day). Once the horizontal heating field has been decomposed into its Fourier components, $J_{k}^{\omega}(\mu)$, for each latitude, the Hough projections are obtained from

$$
Q_{k n}^{\omega}=\int_{-1}^{1} J_{k}^{\omega}(\mu) \Theta_{k n}^{\omega}(\mu) d \mu .
$$

Hough projections were performed for each manifold of the Hough modes (eastward and westward gravity modes and Rossby modes) at frequencies up to $4 \mathrm{cpd}$ and zonal wavenumbers $k \leq 128$. These limits correspond to a range of meridional wavenumbers $0<n-$ $k<180$. Although GCI data have a zonal resolution of $k=256$ (512 pixels for each latitude), we have restricted our analysis of Hough projections to zonal wavenumbers up to 128 . Even so, the zonal resolution of the GCI is twice that of CCM3, since, as noted earlier, the zonal resolution for the heating field in the latter is $k=64$.

Since we are interested in waves that can propagate into the middle atmosphere, we restrict our analysis to vertical wavelengths between 5 and $100 \mathrm{~km}$. Shorter waves are not forced efficiently by the assumed heating profile (23) and, under most circumstances, their intrinsic frequency and vertical group velocities are so small that they would be dissipated within a few kilometers of the source region. The vertical wavelength restriction selects a range of Hough modes for the horizontal projections because the vertical wavelength is related to the eigenvalues $\varepsilon_{k n}^{\omega}$ by means of the dispersion relationship (13); our selection of vertical wavelengths corresponds to eigenvalues $62<\varepsilon_{k n}^{\omega}<11000$ (and equivalent depth $8 \mathrm{~m}<h<1400 \mathrm{~m}$ ).

Once the heating field has been projected onto the Hough modes, the dynamical response to the forcing is calculated. We will consider the response in the near field, just above the top of the convective source, where the dynamical variables have not yet been influenced by processes like wave refraction and absorption in the stratosphere (Garcia and Salby 1987). For this purpose, we calculate the geopotential projections onto Hough modes using Eq. (15) evaluated at $z=12 \mathrm{~km}$, that is, immediately above the forcing region. Finally, the vertical component of the EP flux is evaluated from (19). 


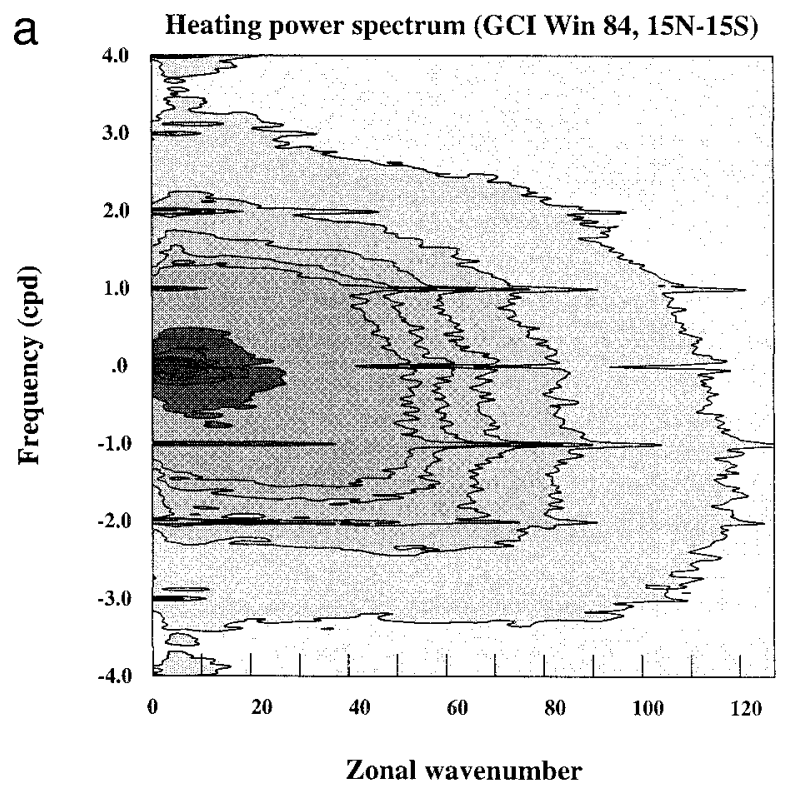

C Heating power spectrum (CCM-Hack Win 84, 15N-15S)

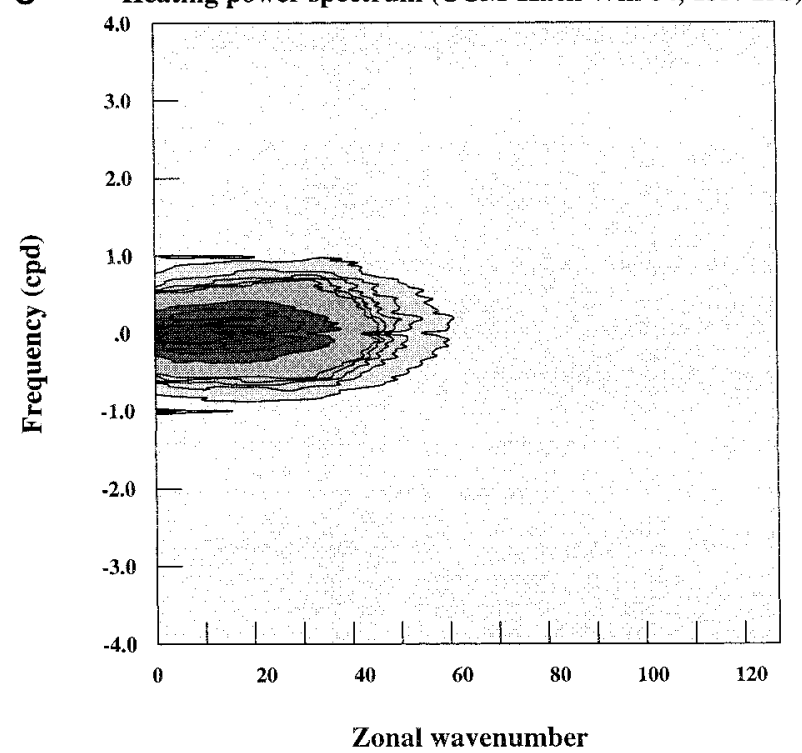

a. Fourier spectra of the heating field

In order to compare the spectra derived from the GCI data to those presented in BS94, we have followed a procedure similar to that used by Bergman and Salby. Furthermore, the conversion from DCA to DCH allows us to compare also the amplitude of the GCI spectra with those obtained from the CCM3 simulations. Hereafter the spectra obtained from GCI observations will be referred to as "GCI" while those obtained from CCM3 will be referred to as "CCM-Z" and "CCM-H," respectively, for the simulations using the ZhangMcFarlane or the Hack parameterizations for convection.

The distribution of variance in both frequency and

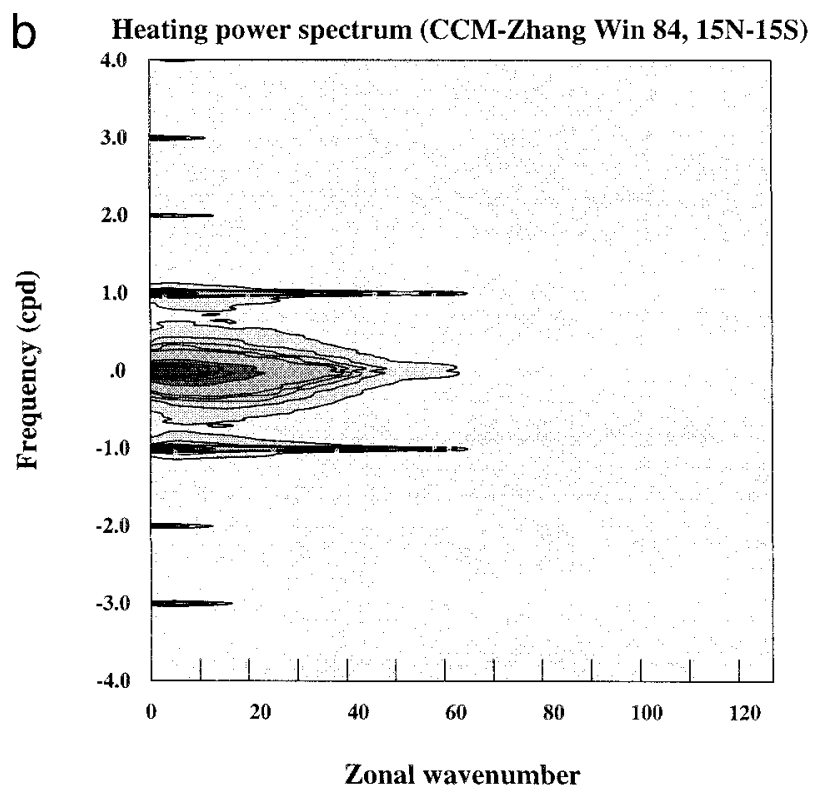

FIG. 7. Frequency-zonal wavenumber spectra of the convective heating for winter 84 obtained from (a) GCI data, (b) CCM-Z, and (c) CCM-H. The spectra are averaged over latitudes $15^{\circ} \mathrm{N}-15^{\circ} \mathrm{S}$ and are normalized to unity at their largest magnitude after applying 8 passes of a 1-2-1 smoother in wavenumber and frequency. Contour increments are linear, but in the region shaded in light gray they are 20 times smaller than in the region shaded in dark gray. Negative and positive frequencies refer to westward- and eastward-propagating waves, respectively.

zonal wavenumber for the GCI and CCM3 results is presented in Fig. 7. Note that the zonal resolution in CCM3 is $k=64$, but the abscissa of the plot extends to $k=128$ in order to facilitate comparison with the plots for GCI data, which have higher spatial resolution. The spectrum of the GCI data shows power smoothly distributed in both frequency and wavenumber, with significant contributions from small scales. The two CCM3 simulations also show power decreasing relatively slowly with zonal wavenumber, but the behavior in frequency is very different from that found in GCI. The spectrum for the CCM-Z simulation (Fig. 7b) is much more "red" in frequency: significant power is found almost exclusively at low frequencies, albeit with large 
peaks at the diurnal frequency and its harmonics. The CCM-H simulation (Fig. 7c) has a smoother distribution of variance in frequency. Nevertheless, power is still concentrated at low frequencies, and in contrast with CCM-Z, variance is very small at the diurnal harmonics.

Figure 8 shows spectra of heating power versus frequency for the GCI and both CCM3 simulations, integrated over all zonal wavenumbers and averaged over latitudes $15^{\circ} \mathrm{N}-15^{\circ} \mathrm{S}$. Time and zonal means are removed and 8 passes of a 1-2-1 smoother are applied. Figure 8a shows that the spectra of convective heating are red, with the largest power at the lowest frequencies. However, the decrease of power versus frequency is much more gradual for the GCI than for either CCM3 simulation. This point is emphasized in Fig. 8b, where the power spectra are shown on a log-log scale. Heating power derived from GCI (solid curve) decreases gradually at higher frequencies, and has prominent diurnal harmonics; high frequencies $(|\omega|>0.5 \mathrm{cpd})$ contribute significantly to the total variance.

The spectrum for CCM-Z (dashed curve) contains much less variance at all frequencies than the GCI spectrum. In fact, except for the very prominent diurnal harmonics, power at most frequencies is insignificant compared to the observations. The spectrum for CCM-H (dot-dashed) is also very red, and at frequencies $|\omega|>$ $0.5 \mathrm{cpd}$, heating power decreases even more rapidly than for CCM-Z. However, the overall power level is much larger for CCM-H, larger even than for the GCI at $|\omega|$ $<0.4 \mathrm{cpd}$ (cf. Table 1). The diurnal components are negligible in the CCM-H spectrum, as already anticipated from the results of the preliminary analysis in Fig. $6 \mathrm{c}$ and from the power spectrum in Fig. 7c, and variance quickly becomes insignificant compared to observations for $|\omega|>0.5 \mathrm{cpd}$.

Consistent with the results of BS94, heating variance derived from GCI data decreases at high frequency according to the power law $|\omega|^{-1.3}$. Although the total heating power for the CCM-Z simulation is much less than that derived from GCI data, its variance at high frequencies decreases only slightly more rapidly, as $|\omega|^{-2}$. On the other hand, the heating spectrum for CCM-H shows the fastest decrease of power at high frequencies, $|\omega|^{-4}$. This sharp decrease of power with frequency also begins at lower frequency $( \pm 0.5 \mathrm{cpd})$ than in CCM-Z or the GCI data.

Figure 8c shows heating power spectra plotted as power times frequency versus the log of frequency. This format emphasizes the high-frequency response without distorting the contribution of individual frequencies to the total variance, which remains proportional to the area under the spectral curve. The figure illustrates the low level of heating power in CCM-Z compared to either the GCI or CCM-H. Note also that the largest heating variance in GCI and CCM-H occurs at frequencies $|\omega|>0.1 \mathrm{cpd}$. This is particularly true of the GCI data, where variance is largest near the diurnal frequency. What little heating variance there is in CCM-Z peaks at $|\omega|<0.1 \mathrm{cpd}$, except for the large peaks at $\pm 1 \mathrm{cpd}$. It is also evident from Fig. 8c that, although the total heating variance deduced from GCI data is comparable to that found in the CCM-H simulation, the distribution of power with frequency is not. In particular, CCM-H variance peaks near $\pm 0.2 \mathrm{cpd}$, where it exceeds the variance of the GCI data by about a factor of 2 . GCI variance, on the other hand, is distributed much more broadly in frequency: almost half of the variance in the GCI data occurs at frequencies between 1 and $4 \mathrm{cpd}$, the Nyquist limit of the observations (cf. BS94).

\section{b. Hough projections of the heating field}

Figure 9 shows the wavenumber-frequency spectrum of the convective heating field derived from GCI data after projection upon the Hough modes, per Eq. (24). The spectrum is obtained by summing over all meridional indices in the three manifolds of the Hough modes. As noted earlier, we are interested in vertically propagating waves that are forced efficiently by the heating profile (23); thus, we consider only modes of vertical wavelength between 5 and $100 \mathrm{~km}$. This restriction leaves out Kelvin and gravity waves with phase speeds of magnitude $|c|=\left|N / m_{\max }\right| \leq 9 \mathrm{~m} \mathrm{~s}^{-1}$. The effect can be seen clearly in the spectrum for GCI data shown in Fig. 9, where the dashed lines refer to zonal phase speeds $c= \pm 9 \mathrm{~m} \mathrm{~s}^{-1}$. The restriction to vertically propagating waves removes much of the low-frequency variance present in the original heating field except at the smallest zonal wavenumbers, as can be appreciated by comparing Figs. 9 and 7a. The same supression of lowfrequency variance occurs for the spectra of CCM-Z and CCM-H after projection onto the Hough modes (not shown).

Figure 10 presents power spectra of the projected heating, $Q_{k n}^{\omega}$ in Eq. (24), as functions of frequency for the GCI data, and the CCM-H and CCM-Z simulations. These spectra are obtained by summing Hough modal contributions over all meridional indices and zonal wavenumbers. Figure 10a displays the results on a linear scale and shows clearly the effect of restricting the Hough projections to vertical wavelengths between 5 and $100 \mathrm{~km}$. As noted above, this restriction alters the

FIG. 8. Frequency spectra of convective heating obtained from GCI data (solid), and the CCM-Z (dashed) and CCM-H (dot-dashed) simulations for winter 84 , averaged between $15^{\circ} \mathrm{N}-15^{\circ} \mathrm{S}$. (a) The spectral density $\left(\mathrm{K}^{2} \mathrm{day}^{-2} \mathrm{cpd}^{-1}\right)$ on a linear scale and (b) on a log-log scale. (c) Spectral density times frequency vs log of frequency (area preserving) in units of $\mathrm{K}^{2}$ day $^{-2}$. Eight passes of a $1-2-1$ smoother were applied. Negative and positive frequencies refer to westward and eastward components, respectively. 
HEATING POWER SPECTRA COMPARISON, (15N-15S)

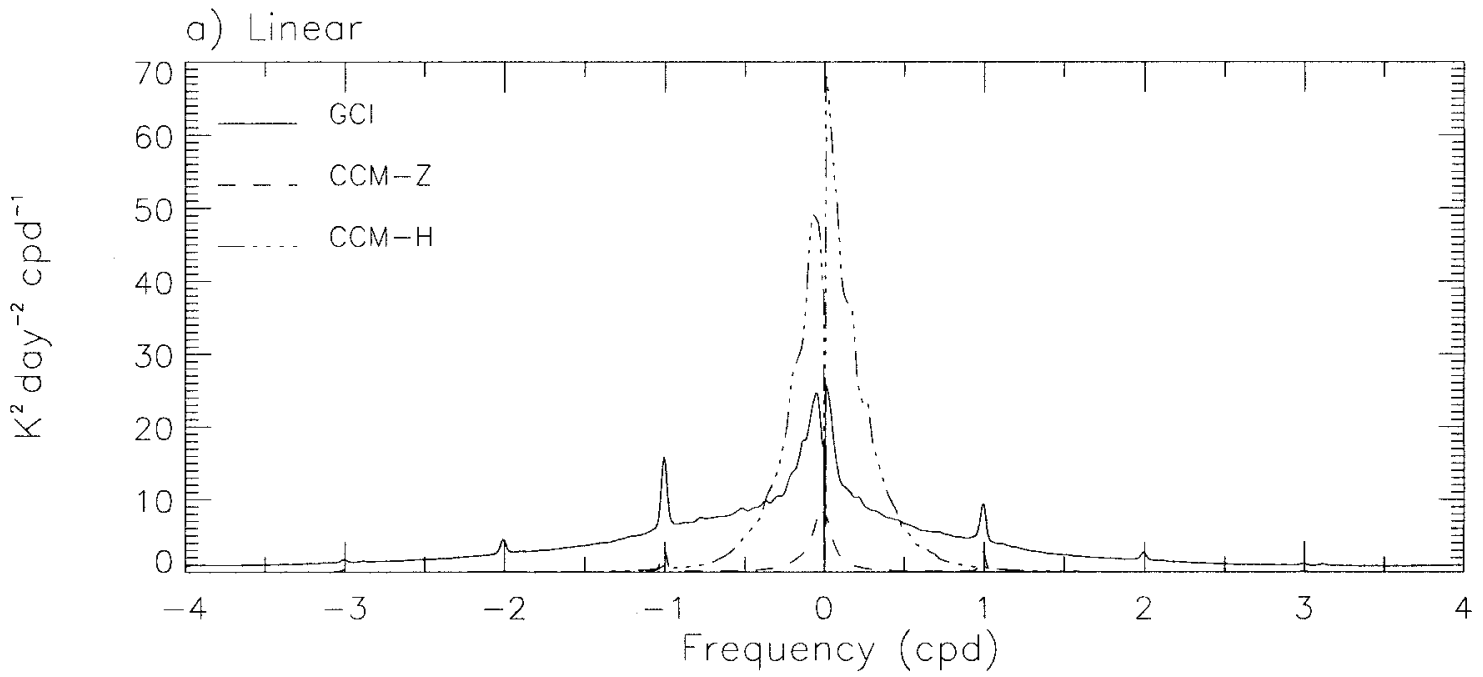

b) $\log -\log$

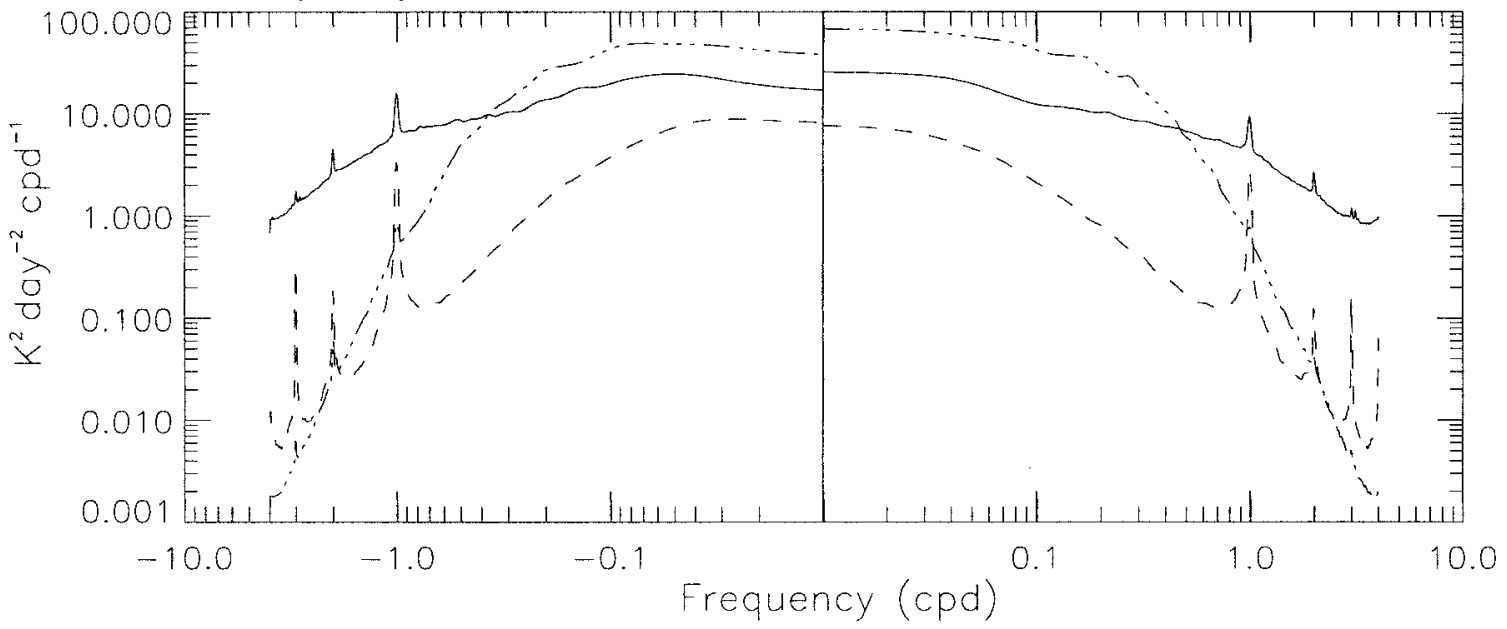

c) |w|*Power spectral density

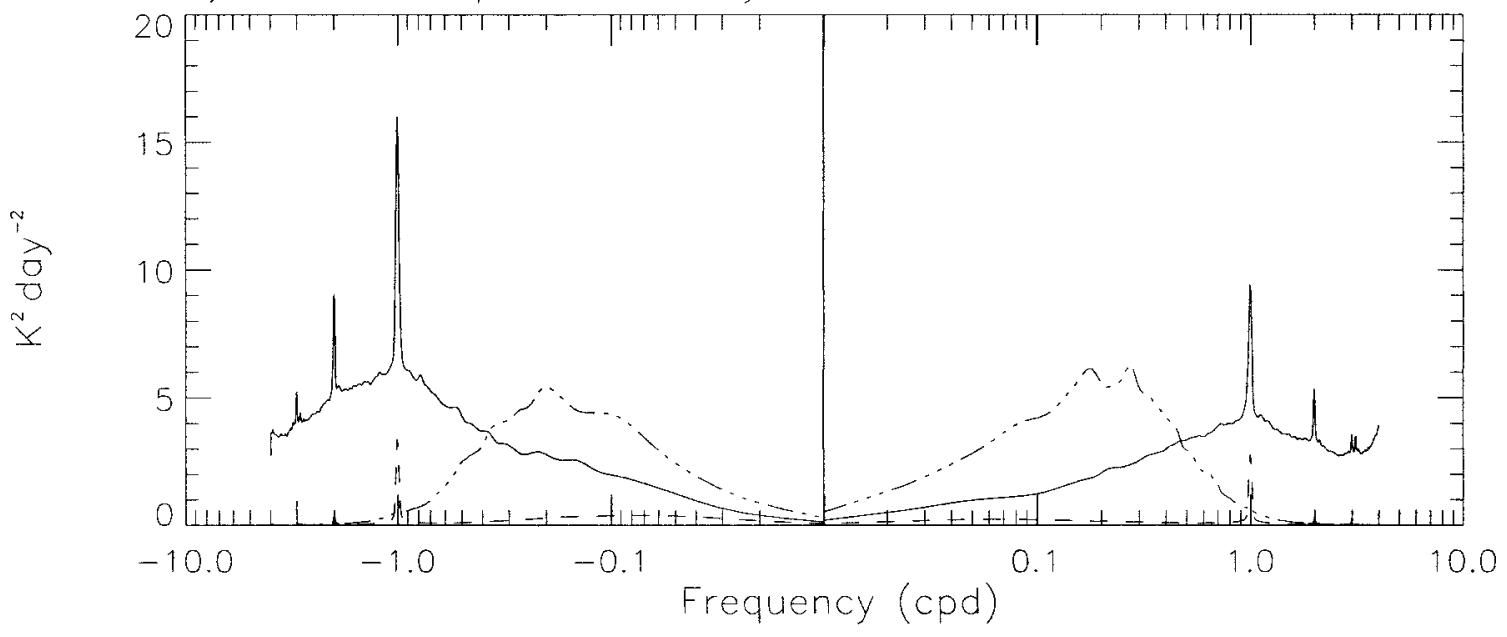




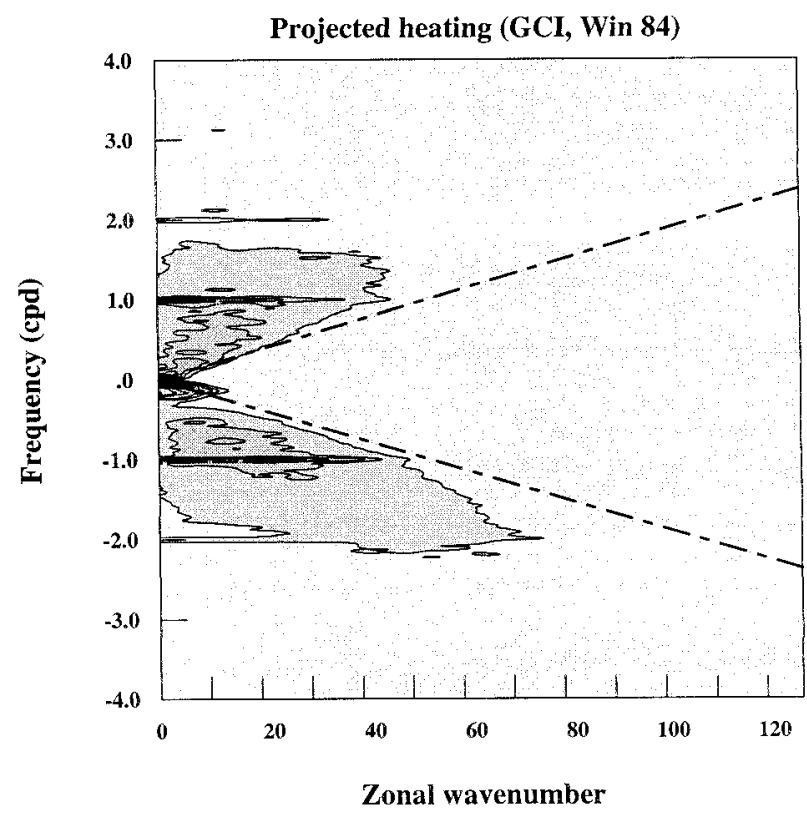

Fig. 9. Frequency-zonal wavenumber spectrum for the Hough projections of the convective heating derived from GCI data, after projection onto Hough modes. The spectrum is obtained by summing the Hough components over the three manifolds and integrating over all meridional indices. It has been smoothed and normalized as in Fig. 7. Contour increments are linear. The dashed lines refer to zonal phase speeds of $\pm 9 \mathrm{~m} \mathrm{~s}^{-1}$; waves with smaller phase speeds do not propagate readily into the middle atmosphere (see text for details).

character of the projected heating spectra: whereas the original (unprojected) heating spectra (Fig. 8a) are red, the projected spectra exhibit a more uniform distribution of power with frequency.

Figure $10 \mathrm{~b}$ shows the spectra on a $\log -\log$ scale, to facilitate comparison among them. The spectra of projected heating for the GCI data and the CCM-H simulation have a distribution of variance that is rather similar at low and intermediate frequencies (i.e., $|\omega|<0.5$ cpd). This result may be compared to the unprojected heating spectra (Fig. 8b), where the CCM-H simulation exhibited substantially larger variance at low frequencies than the GCI data. The spectrum of projected heating for the CCM-Z simulation contains much less total power than either the GCI or the CCM-H spectrum. However, at frequencies $\geq 1 \mathrm{cpd}$, the variance in CCM-Z is comparable to or greater than the variance in $\mathrm{CCM}-\mathrm{H}$ because the latter falls off very rapidly at $|\omega|>0.5 \mathrm{cpd}$. Figure $10 \mathrm{~b}$ also shows a region of very low power in all three spectra at $\omega \simeq-0.3 \mathrm{cpd}$. This corresponds to the "spectral gap" between (low frequency) Rossby waves and (high frequency) westward inertia-gravity waves. Similar behavior is not observed at positive frequencies because Kelvin waves span continuously the range from low to high frequencies.

Although the power of the projected heating appears to be similar for the GCI and CCM-H spectra of Fig. $10 \mathrm{~b}$, displaying the results in variance-preserving format (power times frequency vs log of frequency, Fig. 10c) shows that, in fact, both CCM-H and CCM-Z greatly underestimate heating variance with respect to GCI, except for $0<\omega<0.5 \mathrm{cpd}$, where heating variance is actually larger for CCM-H than for GCI. The discrepancy between CCM-H and GCI in this frequency range apparent in the original heating spectrum (Fig. 8c) is evidently associated with (Rossby) waves that do not propagate efficiently into the middle atmosphere.

\section{c. The vertical component of EP flux, $F_{z}$}

Once the heating field has been projected onto the Hough modes in the range of interest, the dynamical response to the heating can be calculated in terms of the geopotential, following the formulation of section 2. The two-dimensional spectrum for the Hough components of the geopotential (not shown) has a very prominent peak at low frequency, due to the inverse frequency dependence in Eq. (15). At higher frequencies a two-lobe structure emerges, with power localized in two spectral regions corresponding to zonal phase speeds of about 45 and $15 \mathrm{~m} \mathrm{~s}^{-1}$. These preferred ranges of phase speed arise from the vertical scale selection discussed in section 2. For Kelvin waves and high-frequency gravity waves, vertical wavenumber, $m$, is related to phase speed, $c$, according to $c=N / m$. Thus, concentration of variance along the lines $c=45 \mathrm{~m} \mathrm{~s}^{-1}$ and $c=15 \mathrm{~m} \mathrm{~s}^{-1}$ corresponds to Kelvin and highfrequency gravity waves with vertical wavelengths of approximately $2 \Delta z$ (first vertical projection) and $2 \Delta z / 3$ (second vertical projection), respectively.

Similar behavior is apparent in the frequency-zonal wavenumber distribution of the vertical component of the EP flux, $F_{z}$, calculated from Eq. (19). Figure 11a shows the distribution of $F_{z}$ obtained from GCI data, summed over all meridional indices and over the three Hough manifolds. [Note that $F_{z}$ is actually negative at positive (eastward) frequencies; $\left|F_{z}\right|$ is plotted instead to enhance readability of the figure.] Although the distribution of $F_{z}$ is red, like the spectra of projected heating (Fig. 9) and geopotential (not shown), the dependence of $F_{z}$ on $k$ and $m$, per Eq. (19), emphasizes higher zonal wavenumbers and also the second vertical projection response (which occurs at higher vertical wavenumber $m$ than the first projection). Distributions of $F_{z}$ derived from CCM-Z and CCM-H are shown in Figs. $11 \mathrm{~b}$ and $11 \mathrm{c}$, respectively. The two-lobe structure is not particularly clear in either spectrum due to the lack of heating power that can excite vertically propagating waves at intermediate and high frequencies (cf. Fig. 10b).

Figure 12 shows frequency distributions of $F_{z}$ derived from the GCI data, and from the CCM-Z and CCM-H simulations. (The quantity plotted is again $\left|F_{z}\right|$.) The distributions are obtained by summing over Hough manifolds, meridional indices, and zonal wavenumbers. The linear distributions (Fig. 12a) are once again "red," like 
PROJECTED HEATING COMPARISON
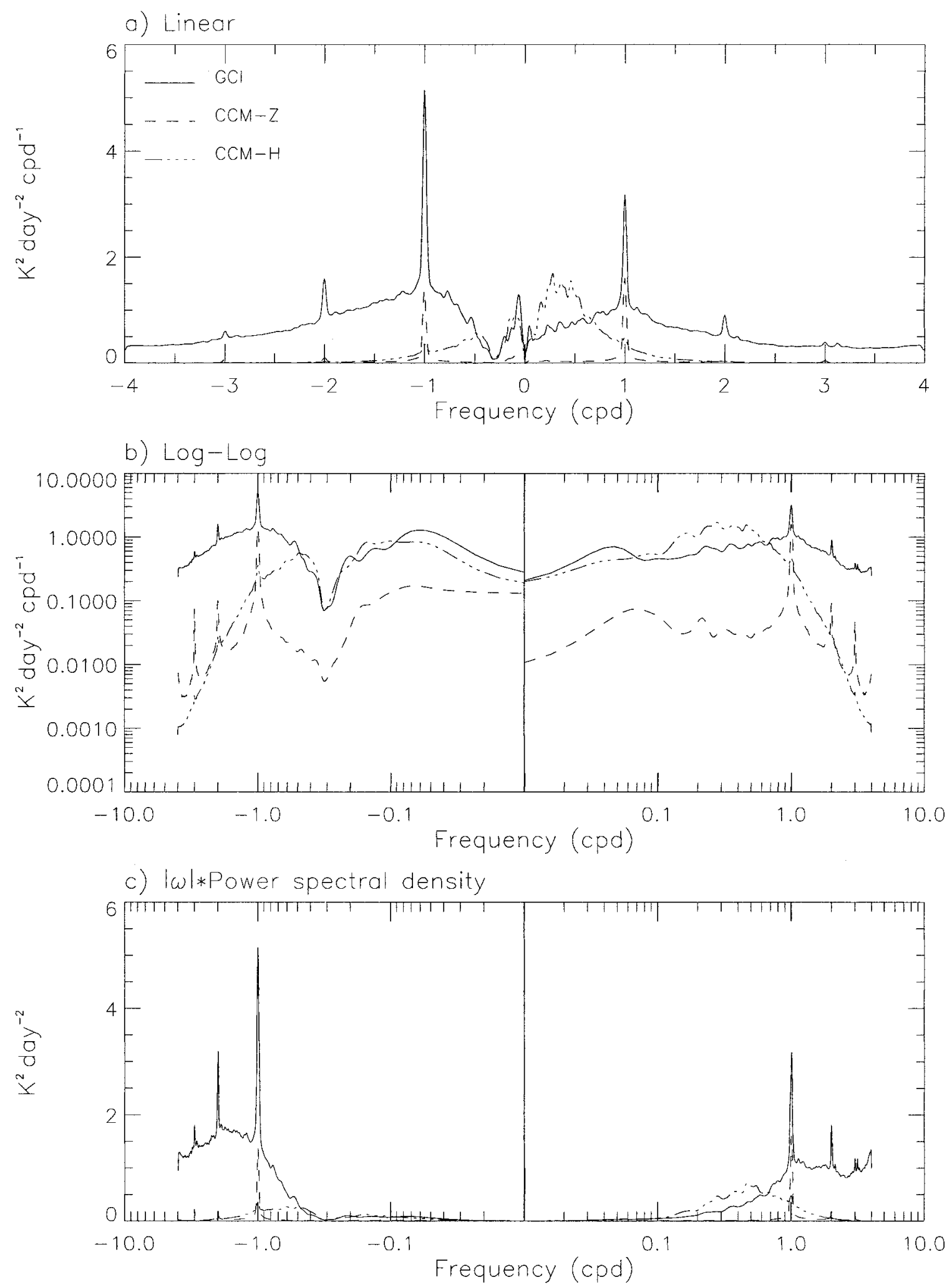

FIG. 10. As in Fig. 8, but for the heating field after projection onto Hough modes. The spectra are obtained by summing over the three Hough manifolds and all meridional indices and zonal wavenumbers. 
a

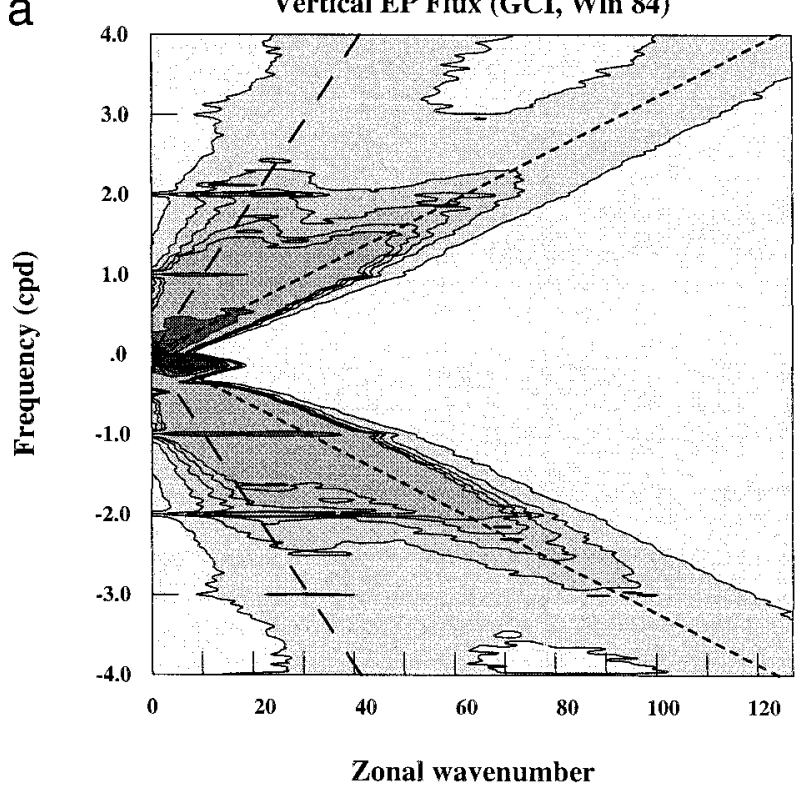

C

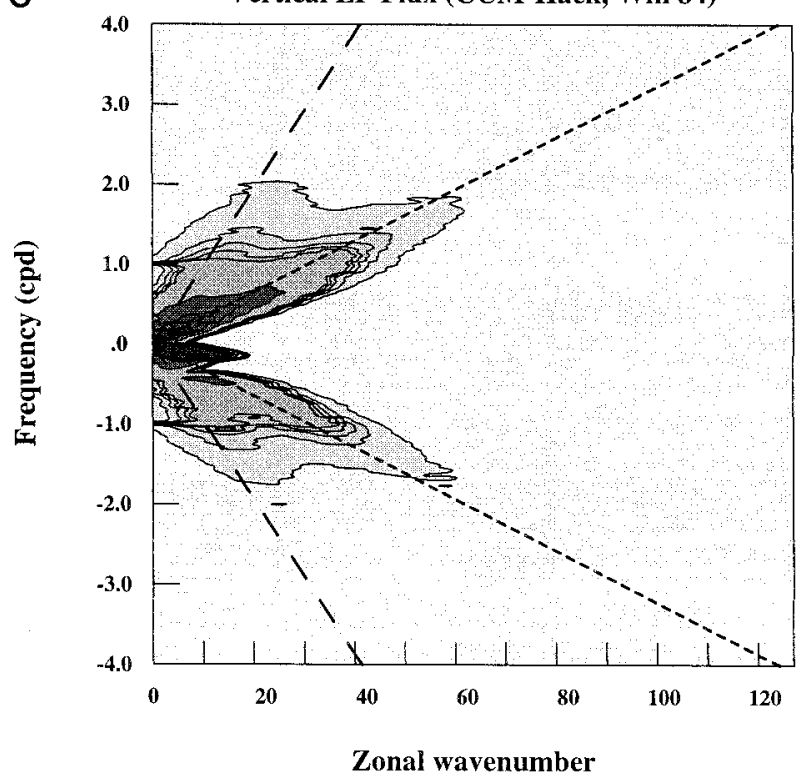

the heating spectrum of Fig. 8a and unlike the projected heating of Fig. 10a.

EP flux distributions in log-log format (Fig. 12b) offer a clearer visualization of the differences between the GCI and the two CCM simulations. The use of the Hack convective scheme (CCM-H) yields a distribution of $F_{z}$ whose variance compares fairly well with the GCI distribution for $|\omega|<0.5 \mathrm{cpd}$, but falls off very rapidly at higher frequencies; the diurnal harmonics are not at all prominent. On the other hand, the Zhang-McFarlane convection scheme (CCM-Z) produces very low $F_{z}$ variance at all frequencies with the exception of the diurnal harmonics. When presented in area-preserving format b

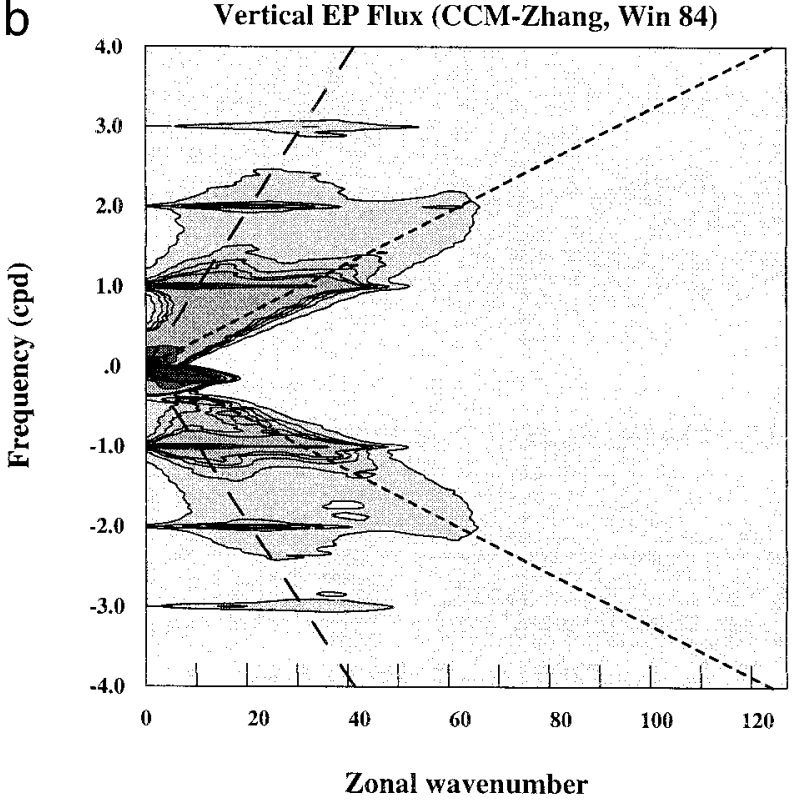

FIG. 11. Frequency-zonal wavenumber distributions of the magnitude of the vertical component of the EP flux, $\left|F_{z}\right|$, obtained from (a) GCI, (b) CCM-Z, and (c) CCM-H. They are obtained by summing over the three manifolds of the Hough modes and over all meridional indices; they are smoothed and normalized as in Fig. 7. The contour increments are linear but reduced by a factor 100 in the region shaded light gray in order to emphasize the high-frequency behavior. The long-dashed and short-dashed lines refer to zonal phase speeds of \pm 45 and $\pm 15 \mathrm{~m} \mathrm{~s}^{-1}$, respectively.

(Fig. 12c) the distribution of $F_{z}$ derived from GCI data shows clearly the large contribution to the total variance from high-frequency waves, behavior that is not present in the distribution of $F_{z}$ derived from either the CCM-Z or the CCM-H simulation. However, at lower frequencies $(\omega \leq 0.5 \mathrm{cpd})$ there is good agreement between the spectra for GCI and CCM-H, although the latter shows more variance than the former at positive frequencies. These frequencies are associated with Kelvin waves, which therefore appear to be forced too strongly by convection in the CCM-H simulation. This is consistent with the findings of Sassi et al. (1993), who showed that Kelvin wave temperature perturbations in CCM2 
VERTICAL COMPONENT OF EP FLUX COMPARISON

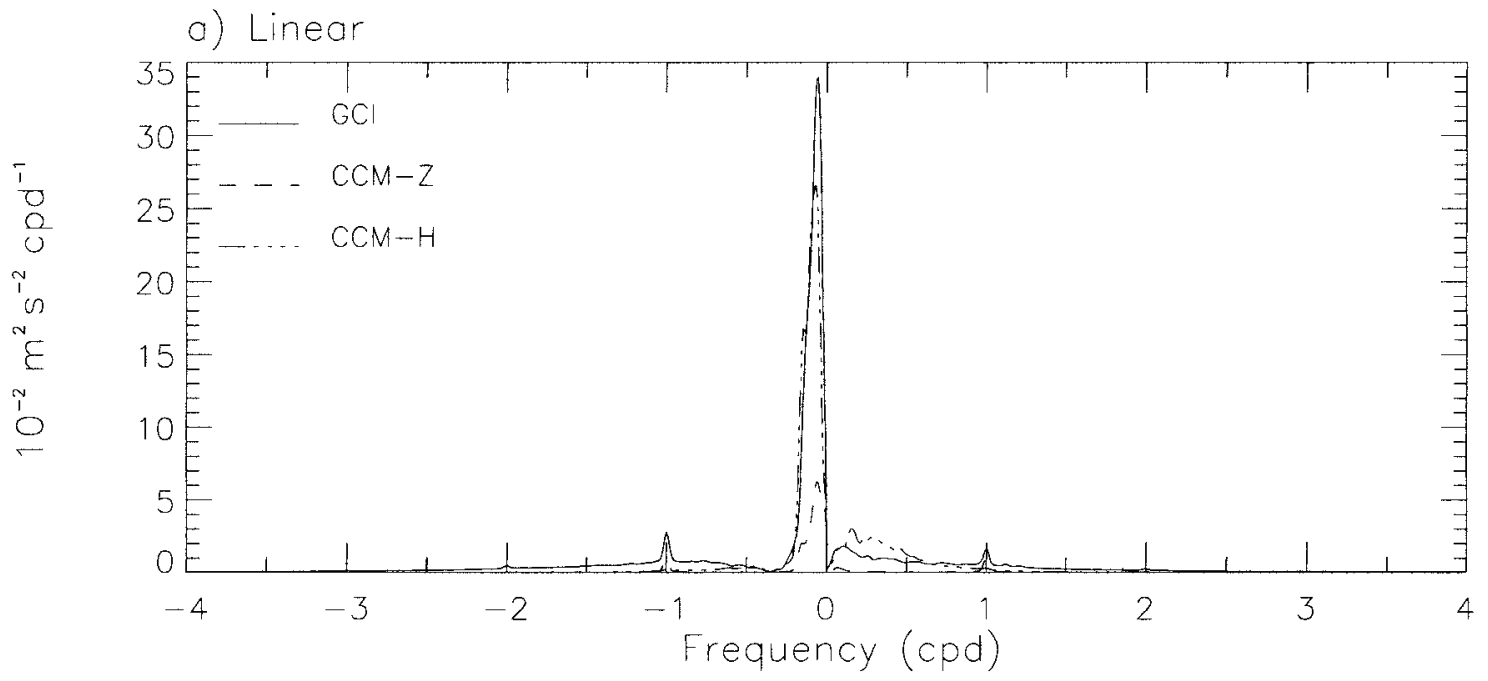

b) $\log -\log$

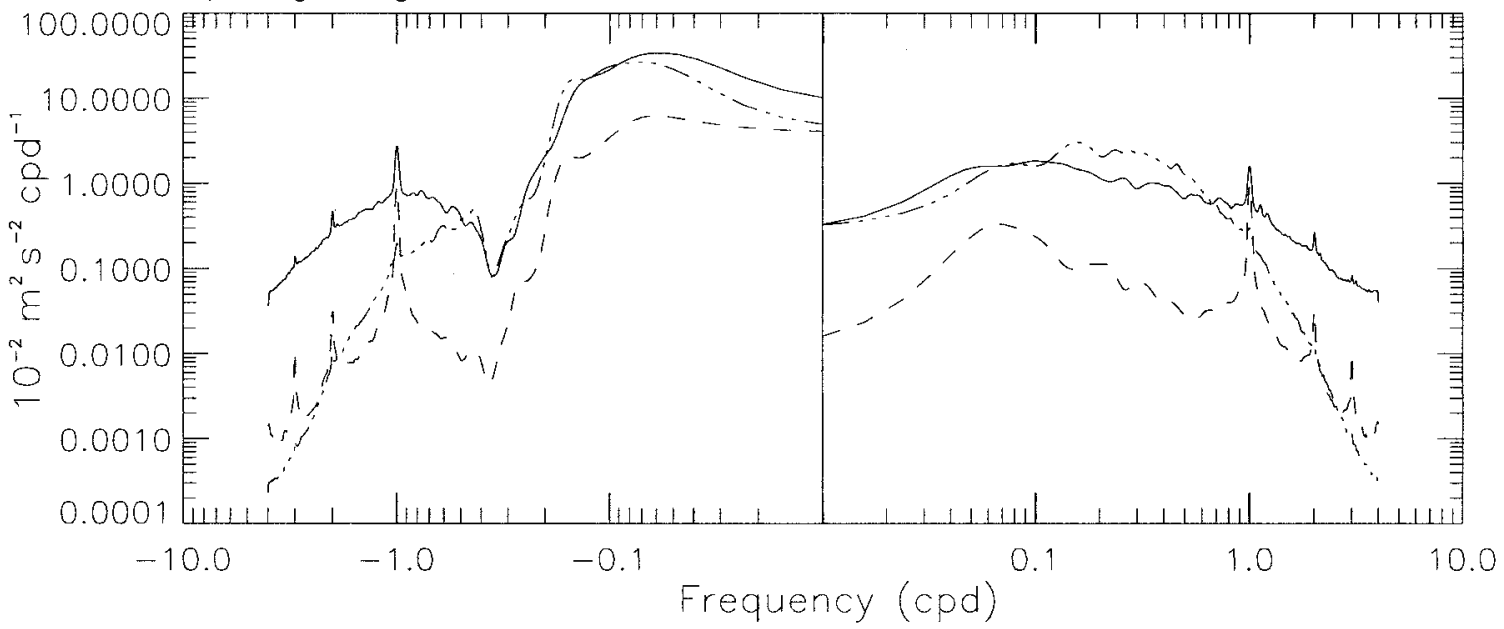

c) $\left|\omega *\left(F_{z}\right)\right|$ spectral density

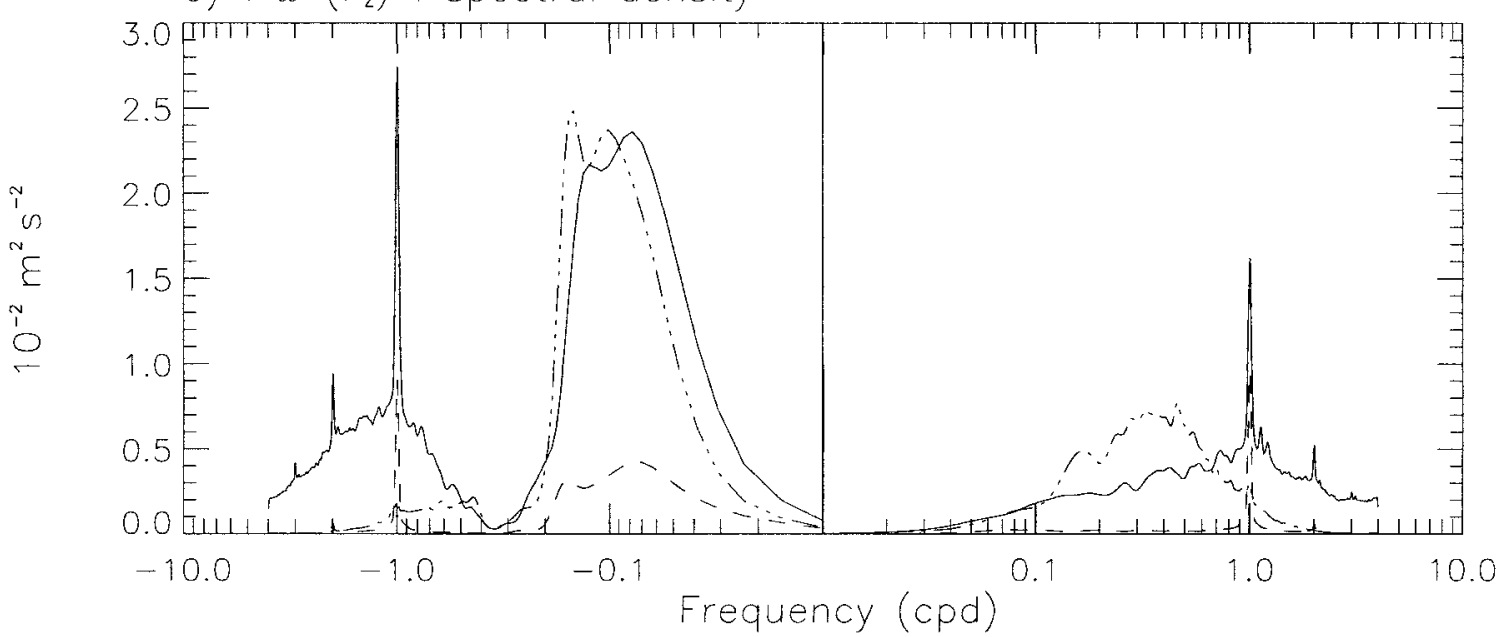

FIG. 12. As in Fig. 8, but for the magnitude of the vertical component of EP flux, $\left|F_{z}\right|$, obtained by summing over zonal wavenumber, Hough manifold, and meridional index. The EP flux is actually positive at westward frequencies and negative at eastward frequencies. Units are $10^{-2} \mathrm{~m}^{2} \mathrm{~s}^{-2} \mathrm{cpd}^{-1}$ for (a) and (b), and $10^{-2} \mathrm{~m}^{2} \mathrm{~s}^{-2}$ for the area-preserving spectrum in (c). 
(which used Hack's convective parameterization) were some $60 \%$ larger than those deduced from satellite observations.

The EP flux distribution for the GCI data shown in Fig. 12c (solid curve) may be compared to the bottom panel of Fig. 14 of BS94. An important difference between our results and those of BS94 is that the contribution to $F_{z}$ from low frequencies is larger in our calculations. This discrepancy is most noticeable for Rossby wave $F_{z}$, which peaks at $\omega=-0.1 \mathrm{cpd}$; it is apparently due to differences in the treatment of thermal dissipation between this study and that of BS94. These differences affect mainly the low-frequency response (i.e., the Rossby waves), since at higher frequencies the influence of thermal damping decreases. Equatorial Rossby waves are not expected to propagate far into the middle atmosphere, so the differences in question do not appear to be particularly relevant to the question of wave forcing of the tropical middle atmosphere.

\section{d. Modal contributions to $F$}

A more detailed comparison of equatorial wave forcing inferred from GCI data and the CCM3 simulations can be carried out by analyzing the contribution of different wave modes to the vertical component of EP flux. For this purpose we have broken down the wave response in terms of Rossby, Rossby-gravity, Kelvin, and inertia-gravity waves. This is possible when $F_{z}$ is calculated from Eq. (19) rather than from the definition (18), since in Eq. (19) $F_{z}$ is proportional to the square of the geopotential for each Hough component.

Table 2 shows the total $F_{z}$, as well as the contributions of each type of wave, derived from GCI data and from the two CCM3 simulations. In all cases, total $F_{z}$ carried by westward-propagating waves is substantially larger than that carried by eastward-propagating waves. This is a consequence of the red nature of the forcing spectrum, which emphasizes the low-frequency Rossby waves. However, because Rossby waves occur at very low intrinsic frequencies and phase speeds, they have small vertical group velocity and are strongly damped before they can propagate far above the forcing region. The values of $F_{z}$ derived from GCI and from CCM-H are similar, both as regards magnitude and distribution between westward- and eastward-propagating waves. However, a closer examination reveals that inertia-gravity waves make a much larger contribution to $F_{z}$ in the GCI data than in CCM-H. In CCM-H only $6 \%$ of $F_{z}$ carried by westward-propagating waves and $16 \%$ of that
TABLE 2. Vertical component of EP flux, $F_{2}$, calculated from Eq. (19), for westward- and eastward-propagating waves and percentage contribution to the total due to Rossby, Rossby-gravity, Kelvin, and inertia-gravity waves; $F_{z}$ has units of $10^{-2} \mathrm{~m}^{2} \mathrm{~s}^{-2}$ and is evaluated at $12 \mathrm{~km}$, immediately above the convective heating source.

\begin{tabular}{|c|c|c|c|}
\hline & GCI & CCM-Z & CCM-H \\
\hline \multicolumn{4}{|c|}{ Westward-propagating waves } \\
\hline Rossby & $1.71(71 \%)$ & $0.31(82 \%)$ & $1.44(84 \%)$ \\
\hline Rossby-gravity & $0.11(5 \%)$ & $0.03(8 \%)$ & $0.17(10 \%)$ \\
\hline Gravity & $0.59(24 \%)$ & $0.04(10 \%)$ & $0.10(6 \%)$ \\
\hline Total westward & 2.41 & 0.38 & 1.71 \\
\hline \multicolumn{4}{|c|}{ Eastward-propagating waves } \\
\hline Kelvin & $-0.28(42 \%)$ & $-0.03(43 \%)$ & $-0.43(67 \%)$ \\
\hline Rossby-gravity & $-0.09(14 \%)$ & $-0.01(14 \%)$ & $-0.11(17 \%)$ \\
\hline Gravity & $-0.29(44 \%)$ & $-0.03(43 \%)$ & $-0.10(16 \%)$ \\
\hline Total eastward & -0.66 & -0.07 & -0.64 \\
\hline
\end{tabular}

carried by eastward-propagating waves is associated with inertia-gravity waves; the corresponding values for GCI are $24 \%$ and $44 \%$. If we ignore the contribution of Rossby waves, the EP flux carried by westward-propagating waves in CCM-H is less than half that in GCI. Neglecting Rossby waves also makes the EP flux in GCI about equal for eastward and westward waves, while in CCM-H eastward waves carry roughly twice as much $F_{z}$ as westward waves.

The results shown in Table 2 may be compared with recent determinations of the EP flux carried by Kelvin and gravity waves. Indirect estimates of $\left|F_{z}\right|$ at tropopause level were obtained from equatorial radiosonde data by Sato and Dunkerton (1997); their indirect method yields the sum of $\left|F_{z}\right|$ due to both eastward- and westward-propagating waves. Depending on the shear in the lowermost stratosphere, the estimates range between 0.7 and $6.0 \times 10^{-2} \mathrm{~m}^{2} \mathrm{~s}^{-2}$, which is divided about equally between eastward- and westward-propagating waves. (Thus, $\left|F_{z}\right|$ due to waves propagating in one or the other direction is in the range $0.35-3.0 \times 10^{-2} \mathrm{~m}^{2}$ $\mathrm{s}^{-2}$.) A similar result was obtained by Horinouchi and Yoden (1998) with their aquaplanet model; their estimates at tropopause level (95 mb), averaged between $13^{\circ} \mathrm{N}$ and $13^{\circ} \mathrm{S}$ and excluding Rossby waves, are 2.02.3 and $1.2-1.4 \times 10^{-2} \mathrm{~m}^{2} \mathrm{~s}^{-2}$ for eastward and westward waves, respectively. From Table 2, the corresponding results for the GCI at $12 \mathrm{~km}$ are 0.66 and $2.41 \times$ $10^{-2} \mathrm{~m}^{2} \mathrm{~s}^{-2}$, but the latter value includes the contribution of Rossby waves. If this is ignored, the EP flux of westward-propagating waves is $0.7 \times 10^{-2} \mathrm{~m}^{2} \mathrm{~s}^{-2}$, only slightly larger than for eastward-propagating waves. Furthermore, because our estimates refer to $z=$

FIG. 13. Magnitude of the vertical component of EP flux for waves of phase speed $|c|>30 \mathrm{~m} \mathrm{~s}^{-1}$. Area-preserving spectral densities $10^{-2}$ $\mathrm{m}^{2} \mathrm{~s}^{-2}$ are shown as functions of (a) frequency, (b) zonal wavenumber, and (c) zonal phase speed. The solid curves denote Kelvin waves (for positive values of the abcissa) and Rossby waves (negative values). The dashed curves denote eastward (positive) and westward (negative) inertia-gravity waves. All spectra are smoothed as in Fig. 8. In (a) the largest amplitude is that of the westward diurnal component, but the ordinate is plotted over the range $0 \%-40 \%$ of the maximum amplitude to enhance readability. Results are shown for GCI (blue) and CCM-H (red). 


\section{VERTICAL COMPONENT OF EP FLUX, C > $30 \mathrm{~m} / \mathrm{s}$}

a) | $\omega *\left(F_{z}\right) \mid$ spectral density

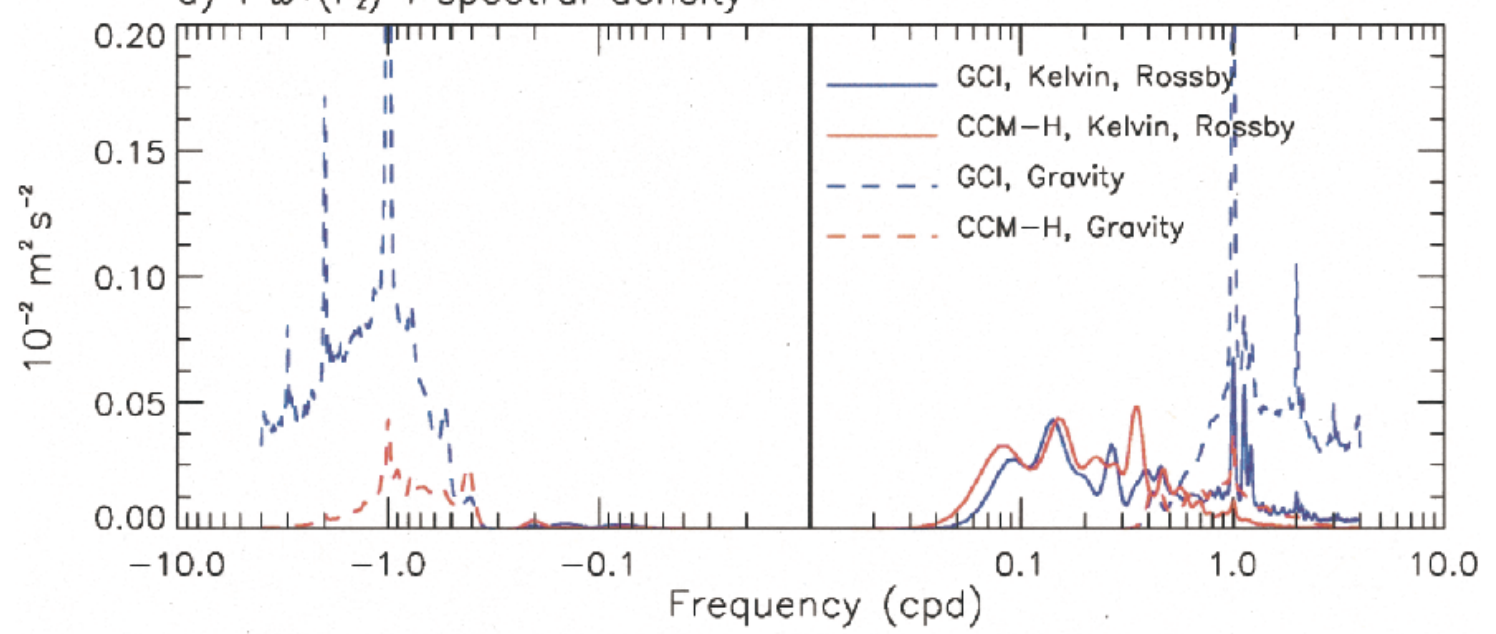

b) | k* $\left(F_{z}\right) \mid$ spectral density

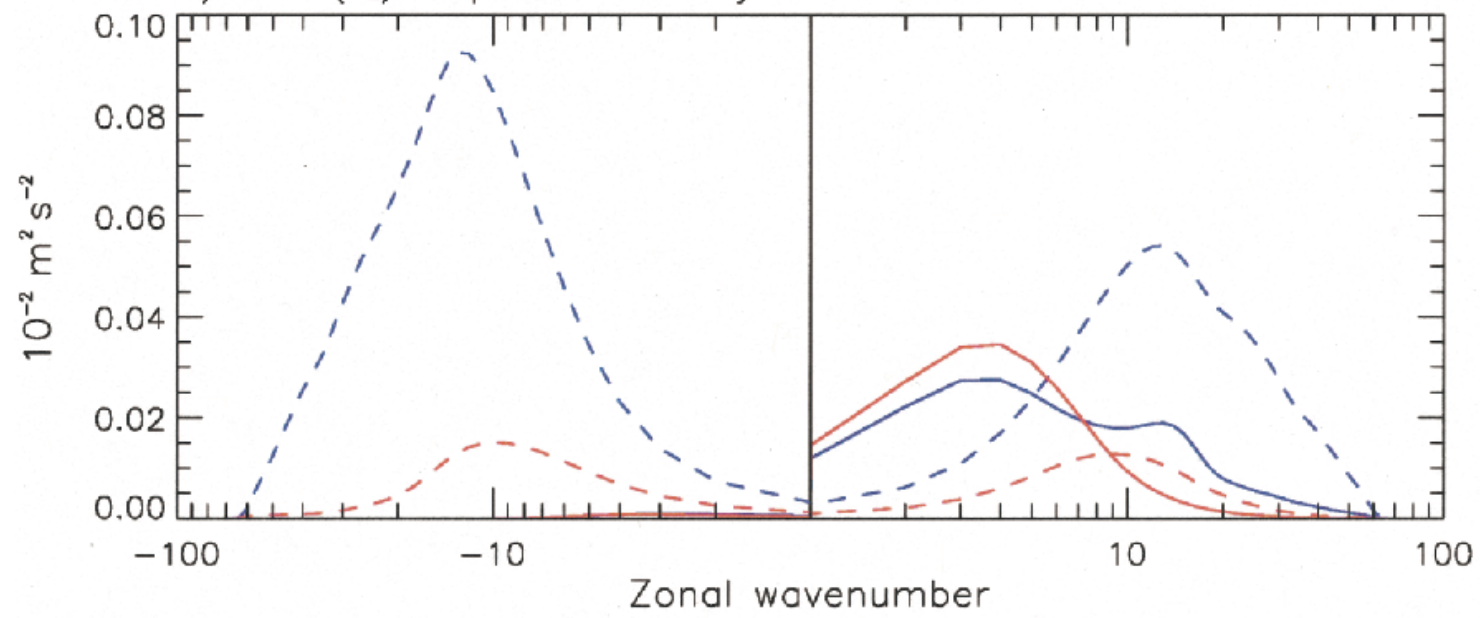

c) $\mid$ c* $F_{z} \mid$ spectral density

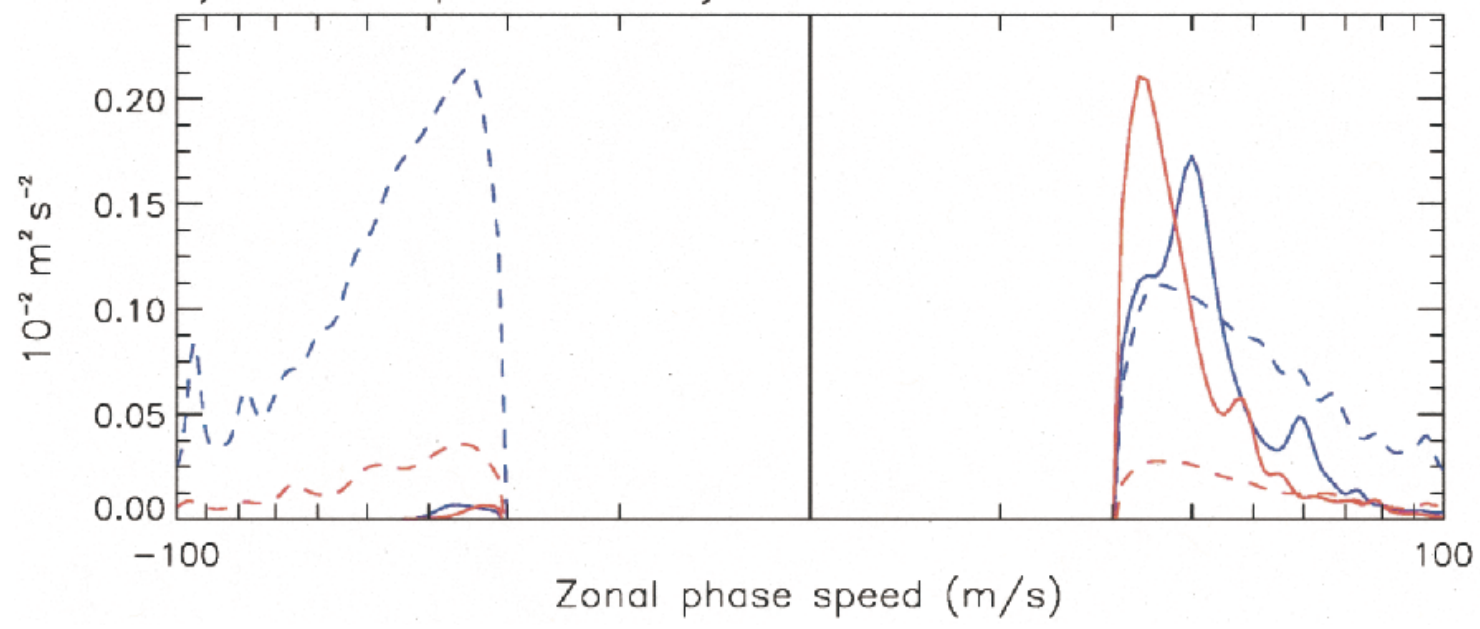


$12 \mathrm{~km}$, about $5 \mathrm{~km}$ below the tropopause, they must be multiplied by a factor of 2 to be comparable to estimates at the tropopause. This yields $\left|F_{z}\right|$ of 1.3 and $1.4 \times 10^{-2}$ for eastward and westward waves, respectively, in reasonably good agreement with the estimates just quoted. The CCM-H results, on the other hand, have a substantially smaller EP flux for westward-propagating waves: the value neglecting the contribution of Rossby waves is $0.27 \times 10^{-2} \mathrm{~m}^{2} \mathrm{~s}^{-2}$ at $12 \mathrm{~km}\left(0.54 \times 10^{-2}\right.$ $\mathrm{m}^{2} \mathrm{~s}^{-2}$ at tropopause level).

As already noted, $F_{z}$ values derived from CCM-Z are much smaller than those obtained from either the GCI or CCM-H (by factors of about 5 and 10, for westwardand eastward-propagating waves, respectively). This reflects the very low level of heating variability present in CCM-Z (cf. Fig. 10). Interestingly, inertia-gravity waves carry a larger fraction of $F_{z}$ in CCM-Z than in CCM-H. In fact, the fraction of $F_{z}$ due to eastwardpropagating gravity waves in CCM-Z (43\%) is almost identical to that in GCI (44\%), although the absolute magnitude is much smaller. This $F_{z}$ is very strongly concentrated at the diurnal period which, as seen earlier, is practically the only source of variability at high frequencies in CCM-Z.

\section{Implications for the forcing of the tropical oscillations}

The foregoing analyses reveal profound differences between the spectrum of vertically propagating waves forced by observed convection (GCI) and that produced by either of the CCM3 simulations. In the case of $\mathrm{CCM}-\mathrm{Z}$, there is a dearth of heating variance, and hence very weak wave forcing, at practically all frequencies. One would expect that the misrepresentation of wave forcing in CCM-Z would affect adversely the simulation of the tropical quasi-biennial and semiannual oscillations. This expectation cannot be verified directly because the two CCM3 simulations discussed in this paper were performed with the standard model, whose upper boundary is located at $3 \mathrm{mb}$, and whose resolution in the stratosphere is too coarse to represent properly the wave field in the Tropics in any case. Unpublished calculations with a middle-atmosphere version of CCM-Z (whose upper boundary is located at $80 \mathrm{~km}$ ) do not reproduce the $\mathrm{QBO}$, or even the $\mathrm{SAO}$, unless a parameterization of small-scale gravity waves is used (F. Sassi 1999, personal communication). This is not at all surprising given that $F_{z}$ in CCM-Z is some 5-10 times smaller than deduced from observations (cf. Table 2).

$\mathrm{CCM}-\mathrm{H}$, on the other hand, displays heating variability comparable to GCI but distributed rather differently in frequency, with most of the variance occurring at periods greater than 2 days. As a result, high-frequency waves, in particular gravity waves, are not forced efficiently in CCM-H. CCM-H has not been run as a middle-atmosphere model; however, version 2 of the Community Climate Model (CCM2), which em- ployed the Hack parameterization, did not produce a QBO or a wholly satisfactory SAO (Sassi et al. 1993). In what follows we compare further the distribution of $F_{z}$ generated by CCM-H and that inferred from GCI in an attempt to elucidate the implications for the forcing of these oscillations. We focus, in turn, on waves expected to play a role in the SAO and the QBO.

\section{a. The semiannual oscillation}

Figure 13 shows distributions of $F_{z}$ plotted in areapreserving format as functions of frequency, $\omega$, zonal wavenumber, $k$, and zonal phase speed, $c$. The plots display $F_{z}$ only for waves of phase speed $|c|>30 \mathrm{~m}$ $\mathrm{s}^{-1}$, since slower waves are unlikely to play a role in the SAO. (Zonal wind extremes associated with the SAO in both the stratosphere and mesosphere are stronger than $30 \mathrm{~m} \mathrm{~s}^{-1}$ —cf. Sassi and Garcia (1997); in addition, the small intrinsic frequencies and vertical group velocities of slower waves imply substantial absorption in the lower and middle stratosphere.) Results derived from GCI are shown in blue, while those obtained from CCM-H are shown in red. The solid curves refer to (eastward propagating) Kelvin waves and (westward propagating) Rossby waves; the dashed curves denote eastward- and westward-propagating gravity waves. The contribution of Rossby waves is barely noticeable in the range of phase velocity considered.

Figure 13a shows that, compared to the GCI observations, CCM-H underestimates severely the EP flux carried by high-frequency gravity waves, while the flux associated with Kelvin waves is roughly comparable for the observations and the model. However, Figs. 13b,c indicate that in CCM-H the EP flux due to Kelvin waves occurs at smaller wavenumbers and phase speeds than in GCI. In fact, the Kelvin-wave $F_{z}$ derived from GCI exceeds that in CCM-H for wavenumbers $k \geq 8$ and phase velocities faster than about $37 \mathrm{~m} \mathrm{~s}^{-1}$. This is a result of stronger forcing around the diurnal frequency in GCI. Sassi and Garcia (1997) have shown that eastward-propagating Kelvin and gravity waves of intermediate scales can play a major role in the westerly phase of the stratospheric SAO. The fact that these waves are not forced efficiently by the Hack convective parameterization may help explain why CCM2 does not produce an entirely satisfactory stratospheric SAO. Presumably, a middle-atmosphere version of CCM-H would also fail to simulate a completely realistic SAO in the stratosphere.

To our knowledge, no GCM has succeeded in simulating the mesospheric SAO. To a large extent this may be due to the placement of the upper boundary. For example, in all middle-atmosphere versions of the NCAR CCM the upper boundary has been located near $80 \mathrm{~km}$, that is, at the altitude where the observed mesospheric SAO achieves maximum amplitude. However, it is unlikely that a model that does not force gravity waves efficiently would produce a mesospheric SAO 
even if the top boundary were placed at a higher altitude. As shown in Fig. 13, a large fraction of $F_{z}$ at eastward phase velocities (and virtually all at westward phase velocities) is carried by gravity waves. CCM-H does not force these waves strongly and thus cannot be expected to simulate the mesospheric SAO even if the model domain were extended well into the lower thermosphere.

\section{b. The quasi-biennial oscillation}

Figure 14 compares the magnitude of $F_{z}$ for waves of phase speed $|c|<30 \mathrm{~m} \mathrm{~s}^{-1}$, that is, waves that are likely to play a role in the stratospheric QBO. In this range of phase speed, Rossby waves are forced strongly and their EP flux is considerable immediately above the source (Table 2). However, Rossby waves have such small intrinsic frequencies and phase speeds that they are unlikely to propagate deeply into the stratosphere. If we consider only gravity waves, it is clear that CCM-H underestimates $F_{z}$ for westward-propagating waves with respect to GCI. Westward gravity waves carry significant $F_{z}$ over a much greater range of wavenumber in GCI than in CCM-H; the total $F_{z}$ of westward gravity waves is also several times larger in GCI than in CCM-H. Note that this $F_{z}$ is carried almost exclusively by waves of zonal wavenumber $k \geq 10$. Indeed, if the contribution of Rossby (and Rossby-gravity) waves is ignored, planetary-scale waves carry negligible EP flux at westward frequencies. At first glance this conclusion may seem at odds with Horinouchi and Yoden's (1998) estimate that $8 \%$ of the $F_{z}$ of westwardpropagating waves is due to wavenumbers $k=1-3$ (see their Table 2). However, these authors considered all phase velocities, while the results shown in Fig. 14 are for $|c|<30 \mathrm{~m} \mathrm{~s}^{-1}$. If faster waves are taken into account, it is clear from Fig. 13 that planetary-scale, westwardpropagating gravity waves do carry a small but significant fraction of the total $F_{z}$.

At eastward frequencies, the EP flux derived from GCI data is comparable to that in CCM-H. Although CCM-H underestimates the forcing of eastward-propagating gravity waves with respect to GCI, that deficit is compensated by the fact that Kelvin waves are forced more strongly in CCM-H than in GCI. Furthermore, because Kelvin waves can occur at much higher frequencies and zonal phase speeds than Rossby waves, their contribution to the QBO should be important throughout the stratosphere. Kelvin waves and eastward-propagating gravity waves do differ in that, while the former contribute to $F_{z}$ at zonal wavenumbers less than about $k=25$, the latter carry $F_{z}$ at considerably larger zonal wavenumbers (Fig. 14b).

In fact, our calculations of $F_{z}$ from GCI data underestimate the total flux carried by the smaller-scale gravity waves at slow phase speeds because we did not use the full spatial resolution of the GCI, but instead truncated it to 128 zonal wavenumbers. Comparison of Figs. $13 \mathrm{~b}$ and $14 \mathrm{~b}$ shows that, for the faster gravity waves, $\left|k F_{z}\right|$ peaks around $k=12$ and decreases rapidly beyond, but for the slower gravity waves, it peaks at $k=30$ and is not negligible even at $k=100$. BS94 (their Fig. 16) compare contributions to $F_{z}$ for different ranges of wavenumber and show that, especially for phase speeds in the range $10<|c|<20 \mathrm{~m} \mathrm{~s}^{-1}$, a substantial fraction of the total $F_{z}$ is carried by gravity waves of $k>60$. These waves are associated with the second vertical projection response, as can be appreciated from Fig. 11a, which also shows how slowly their contribution to $F_{z}$ decreases with $k$. At these large wavenumbers, the waves cannot be readily identified as individual equatorial modes (although they can still be represented formally in terms of the Hough decomposition employed in this study); it is better to think of this broad spectrum at high zonal wavenumbers as representing a continuum of small-scale gravity waves (at $k=60$ the zonal wavelength is about $650 \mathrm{~km}$ ).

In any case, the comparisons presented in Fig. 14 may be interpreted as evidence that small-scale gravity waves play an important role in the stratospheric QBO. Such waves are crucial for driving both phases of the QBO-like oscillation in the model of Horinouchi and Yoden (1998), ${ }^{3}$ although the truncation in their model restricts the zonal wavenumber to $k \leq 42$. The possible importance of gravity waves for the QBO has also been emphasized by Hayashi and Golder (1994). However, the smaller-scale gravity waves alone are probably not sufficient to drive the QBO; detailed understanding of the QBO is likely to involve assessment of the combined effect of waves over a broad range of wavenumbers and frequencies (Dunkerton 1997). Further investigation is also needed on the influence of coarse vertical resolution and of numerical "hyperdiffusion" (see Horinouchi and Yoden 1998). Finally, the role of the background zonal wind in the vicinity of the forcing region also bears examination. BS94 have shown that waves associated with the second projection response are quite sensitive to the background wind; a westerly (i.e., eastward) wind as small as $-5 \mathrm{~m} \mathrm{~s}^{-1}$ can enhance substantially the westward wave response while supressing the eastward waves, while the opposite effect occurs when the background wind is easterly (westward). Although a background zonal wind was not included in our calculations, its effects would not affect materially the foregoing conclusions, which depend upon the very large differences in the power spectra of observed versus modeled convective heating.

\footnotetext{
${ }^{3}$ We have calculated the excitation of equatorial waves by the convective heating from the model of Horinouchi and Yoden; heating time series were kindly provided by Dr. T. Horinouchi. Despite the simple treatment of convection via moist convective adjustment (or perhaps because of it), this aquaplanet model excites a spectrum of equatorial waves comparable to that inferred from the GCI data.
} 
VERTICAL COMPONENT OF EP FLUX, $\mathrm{C}<30 \mathrm{~m} / \mathrm{s}$

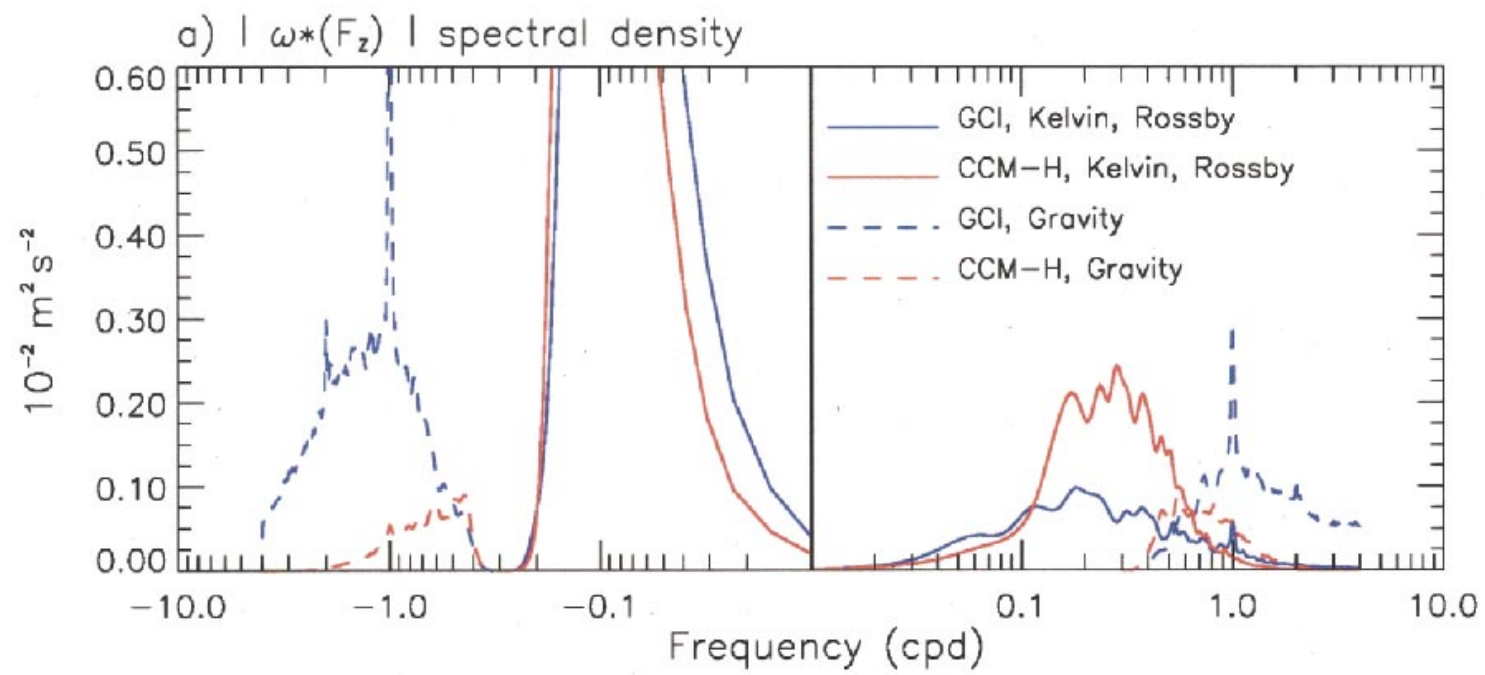

b) I k* $\left(F_{z}\right) \mid$ spectral density

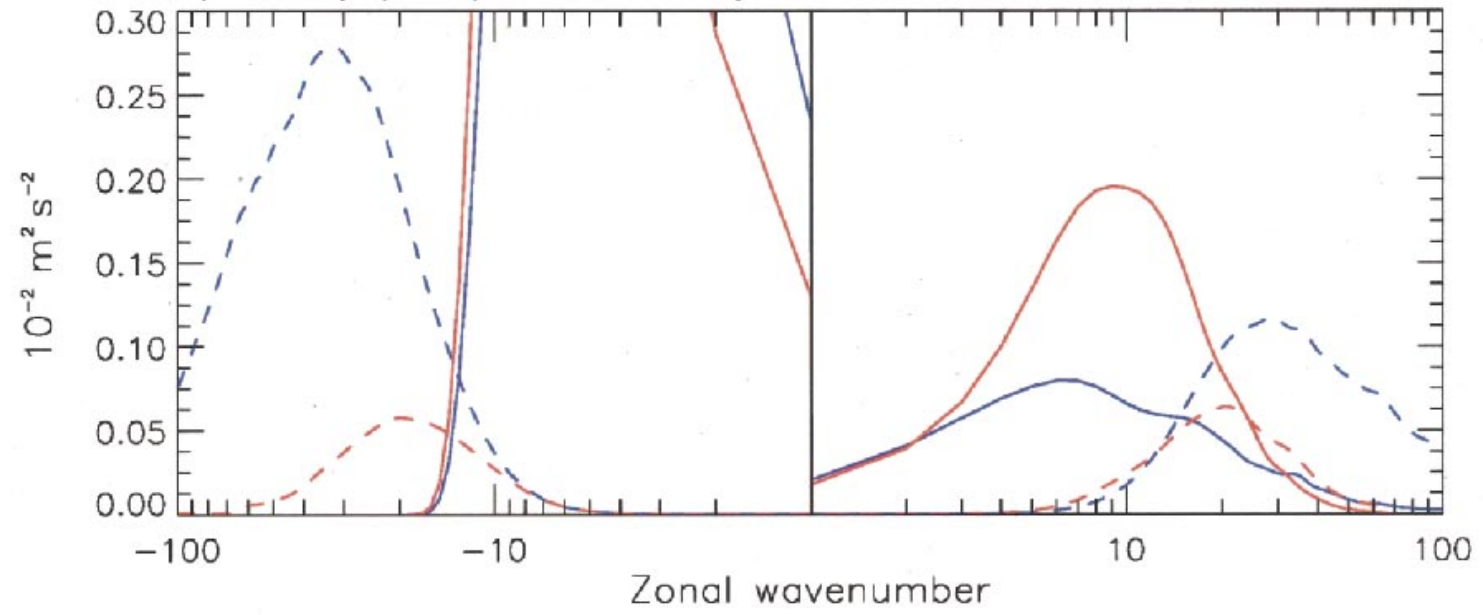

c) $\mid$ c* $F_{z} \mid$ spectral density

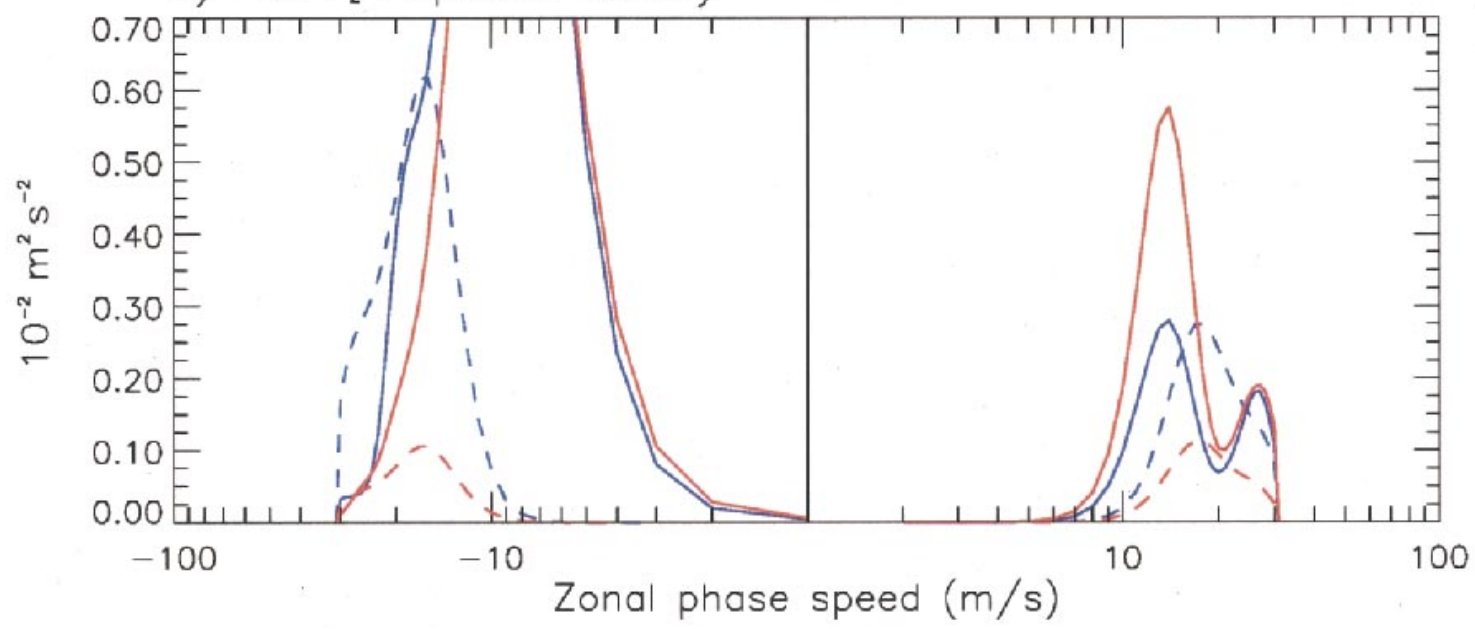

FIG. 14. As in Fig. 13, but for waves of phase speed $|c|<30 \mathrm{~m} \mathrm{~s}^{-1}$. The largest $\left|F_{z}\right|$ in each case is due to Rossby waves; in all panels the ordinates are plotted over the range $0 \%-40 \%$ of the maximum amplitude to enhance readability. 


\section{Summary and conclusions}

We have analyzed the spectra of convective heating from two simulations of the NCAR Community Climate Model (CCM3), and from observations of convective clouds in the Tropics. The excitation of waves by convective heating was evaluated using the method of Bergman and Salby (1994) and characterized in terms of the vertical component of EP flux, $F_{z}$. The two versions of CCM3 considered differed only in the parameterization used to represent convective adjustment. One of the simulations, CCM-Z, used the scheme of Zhang and McFarlane (1995), which is the current standard for CCM3. The second simulation, CCM-H, used the Hack (1994) parameterization (which was the main convective scheme in the previous version of the Community Climate Model, CCM2).

Both simulations reproduce reasonably well the distribution and time mean magnitude of convective heating; however, there are large differences between the variability of the heating in the model and that inferred from observations. These differences are reflected in the EP flux carried by vertically propagating waves. In $\mathrm{CCM}-\mathrm{Z}, F_{z}$ is severely underestimated at all frequencies, except for the diurnal harmonics (which are nonetheless weaker than inferred from observations). The EP flux calculated with CCM-H compares reasonably well with that inferred from observations at frequencies $|\omega|<0.5$ cpd, but falls off very rapidly at higher frequencies. The deficit at higher frequencies is due to weak excitation of gravity waves on all spatial scales. As shown in Table 2 , the GCI data imply that gravity waves make a large contribution to the vertical component of EP flux, but both $\mathrm{CCM} 3$ runs underestimate the forcing of these waves.

We have argued in section 5 that inefficient forcing of high-frequency waves can have important consequences for the simulation of the zonal wind oscillations of the tropical middle atmosphere. It is almost certainly responsible for the failure of GCMs to produce a mesospheric SAO, or a wholly realistic stratospheric SAO. It probably accounts in part for the almost universal inability of GCMs to simulate the QBO, although other factors such as spatial resolution and numerical diffusion are also likely to play a role. A QBO-like oscillation has recently been obtained with an "aquaplanet" model by Horinouchi and Yoden (1998). One major difference between that model and the CCM3 simulations discussed here is the much greater level of high-frequency heating variability in the Tropics. Gravity waves are apparently excited much more efficiently in this model, and to some extent also in the Geophysical Fluid Dynamics Laboratory "SKYHI" model (see, e.g., Manzini and Hamilton 1993), than in either of the versions of CCM3 studied here. Interestingly, very high resolution runs of the SKYHI model also produce QBO-like behavior (K. Hamilton, 1999, personal communication), supporting the idea that the smaller-scale waves play an important role in the oscillation.

Although we have emphasized the impact of transient heating on vertically propagating waves, low levels of heating variance can also affect the simulation of tropical dynamics in the troposphere. The principal mode of intraseasonal variability in the troposphere is the Madden-Julian oscillation (MJO; Madden and Julian 1994), which most GCMs have difficulties in simulating (see Slingo et al. 1995). Analysis of the velocity potential field at $250 \mathrm{mb}$ for the two CCM3 simulations (not shown) reveals a strong MJO signal in CCM-H, while in CCM-Z the signal is considerably weaker (see also Hurrell et al. 1998). Although the convective heating scheme in CCM-Z has major, well-documented virtues when it comes to reproducing the time mean behavior of convection (see, e.g., Zhang et al. 1998; Hack et al. 1998), its very low level of heating variability appears to be a major drawback as regards the simulation of wave motions in the Tropics.

The foregoing considerations point to the central role of convection in the dynamics of the tropical atmosphere. In a time mean sense, convection redistributes heat and drives the Hadley and Walker cells; in addition, the unsteadiness of convection is the major excitation mechanism for transient motions in the Tropics. Because convection involves space scales that cannot be resolved in GCMs, it must be parameterized. Parameterizations of convection have usually been evaluated in terms of their ability to reproduce faithfully the time mean distribution of tropical convection, and this remains a principal desideratum. However, our results suggest that the variability of convection must also be captured if largescale models are to simulate accurately the transient components of the tropical circulation.

Acknowledgments. The authors wish to thank Brian Eaton for his help in carrying out the CCM calculations. They also are grateful to John Bergman, Byron Boville, James Hack, Murry Salby, and Anne Smith for helpful comments and discussions. Dr. Takeshi Horinouchi kindly provided convective heating data from the AGCM simulation described in Horinouchi and Yoden (1998). L.R. was supported in part by the Atmospheric Chemistry Division and the Advanced Study Program of NCAR, and by Universitá dell'Aquila (Italy).

\section{APPENDIX}

\section{Conversion from Deep Convective Activity (DCA) Index to Deep Convective Heating (DCH) Rate}

For a uniform vertical distribution of heating, the heating term due to condensation of water vapor can be written as

$$
\frac{J}{c_{p}}=\frac{1}{c_{p}} L_{c}(C-E) \approx \frac{1}{c_{p}} L_{c}\left(\frac{m_{w}}{\rho_{w} \Delta z}\right),
$$


where $m_{w}$ is the average mass of water that condenses per unit time for a column of depth $\Delta z$ and unit surface area, and $\rho_{w}$ is the density of water. It follows that the ratio $m_{w} / \rho_{w}$ represents the volume of condensed water that precipitates per unit surface area. If we denote this ratio by $\mathcal{P}\left(\mathrm{mm} \mathrm{day}^{-1}\right)$, then (A1) can be written as

$$
\frac{J}{c_{p}} \approx \frac{L_{c}}{c_{p} \Delta z} \mathcal{P},
$$

where $J / c_{p}$ has units of $\mathrm{K} \mathrm{day}^{-1}$ and $\mathcal{P}$ has units of $\mathrm{mm}$ day $^{-1}$.

The latent heat of condensation for water is $L_{c}=2.5$ $\times 10^{6} \mathrm{~J} \mathrm{~kg}^{-1}$ and $c_{p}=1.004 \times 10^{3} \mathrm{~J} \mathrm{~kg}^{-1} \mathrm{~K}^{-1}$. For the depth of the convective column we take $\Delta z=10.3$ $\mathrm{km}$, which is consistent with the depth of the convective heating assumed in our calculations [see Eq. (23) and related discussion in section 3]. This choice yields

$$
\frac{J}{c_{p}}=0.24 \mathcal{P},
$$

where again $J / c_{p}$ is in $\mathrm{K} \mathrm{day}^{-1}$ and $\mathcal{P}$ in $\mathrm{mm} \mathrm{day}^{-1}$.

The DCA index defined in section 3 can be related to the observed precipitation. Hendon and Woodberry (1993) obtained a conversion factor from DCA to precipitation by comparing the mean precipitation observed in the tropical region $\left(21^{\circ} \mathrm{N}-21^{\circ} \mathrm{S}\right)$ during the years 1986-89 (4 mm day ${ }^{-1}$, Janowiak and Arkin 1991) to the mean DCA for June 1983-June 1984 (0.57 DCA units). They thus found $P\left(\mathrm{~mm} \mathrm{day}^{-1}\right)=7.02$ DCA. However, Hendon and Woodberry used a temperature threshold of $230 \mathrm{~K}$ to define their DCA index, whereas we use $240 \mathrm{~K}$. With the latter threshold we obtain a mean tropical DCA for the "winter 84" season (see section $3 \mathrm{a})$ of 1.1 units, and so $\mathcal{P}\left(\mathrm{mm} \mathrm{day}^{-1}\right)=3.64$ DCA. Using this value in (A3) we arrive at

$$
\mathrm{DCH} \quad\left(\mathrm{K} \mathrm{day}^{-1}\right)=0.87 \mathrm{DCA} \text {. }
$$

Strictly speaking, the above conversion should be carried out using the mean precipitation rate for the same period as the DCA, that is, the winter 84 season analyzed in this paper. Such data were not available to us, so we have used the mean rate for 1986-89 estimated by Janowiak and Arkin. Thus, Eq. (A4) will be inaccurate to the extent that the mean precipitation rate for winter 84 is different from Janowiak and Arkin's mean. However, Fig. 10 of Janowiak and Arkin's (1991) study suggests that the problem may not be severe, since it shows that seasonal and interannual deviations from the 1986-89 mean tropical precipitation rate are within $10 \%$ of the mean value.

\section{REFERENCES}

Andrews, D. G., J. R. Holton, and C. B. Leovy, 1987: Middle Atmosphere Dynamics. Academic Press, 489 pp.

Arkin, P. A., and P. E. Ardanuy, 1989: Estimating climatic-scale precipitation from space: A review. J. Climate, 2, 1229-1238.

Bergman, J. W., and M. L. Salby, 1994: Equatorial wave activity derived from fluctuations in observed convection. J. Atmos. Sci., 51, 3791-3806.

Boville, B. A., and X. Cheng, 1988: Upper boundary effects in a general circulation model. J. Atmos. Sci., 45, 3015-3034.

, and W. J. Randel, 1992: Equatorial waves in a stratospheric GCM: Effects of vertical resolution. J. Atmos. Sci., 49, 785801.

Dunkerton, T. J., 1985: A two-dimensional model of the quasi-biennal oscillation. J. Atmos. Sci., 42, 1151-1160.

, 1997: The role of gravity waves in the quasi-biennial oscillation. J. Geophys. Res., 102, 26 053-26076.

Elbert, E. E., M. J. Manton, P. A. Arkin, R. J. Allam, G. E. Holpin, and A. Gruber, 1996: Results from the GPCP Algorithm Intercomparison Programme. Bull. Amer. Meteor. Soc., 77, 28752887.

Garcia, R. R., and M. L. Salby, 1987: Transient response to localized episodic heating in the Tropics. Part II: Far-field behavior. $J$. Atmos. Sci., 44, 499-530.

Gray, L. J., and J. A. Pyle, 1989: A two-dimensional model of the quasi-biennal oscillation of ozone. J. Atmos. Sci., 46, 203-220.

Gray, W. M., and R. W. Jacobson, 1977: Diurnal variation of deep cumulus convection. Mon. Wea. Rev., 105, 1171-1188.

Hack, J. J., 1994: Parameterization of moist convection in the National Center for Atmospheric Research Community Climate Model (CCM2). J. Geophys. Res., 99, 5551-5568.

_ J. T. Kiehl, and J. W. Hurrell, 1998: The hydrologic and thermodynamic characteristics of the NCAR CCM3. J. Climate, 11, 1179-1206.

Hayashi, Y., and D. Golder, 1994: Kelvin and mixed Rossby-gravity waves appearing in the GFDL "SKYHI" general circulation model and the FGGE dataset: Implications for their generation mechanism and role in the QBO. J. Meteor. Soc. Japan, 72, 901-935.

- — _ , and J. D. Mahlman, 1984: Stratospheric and mesospheric Kelvin waves simulated by the GFDL "SKYHI" general circulation model. J. Atmos. Sci., 41, 1971-1984.

Heddinghaus, T. R., and A. F. Krueger, 1981: Annual and interannual variations in outgoing longwave radiation over the Tropics. Mon. Wea. Rev., 109, 1208-1218.

Hendon, H. H., and K. Woodberry, 1993: The diurnal cycle of tropical convection. J. Geophys. Res., 98, 16 623-16 637.

Hirota, I., 1978: Equatorial waves in the upper stratosphere and mesosphere in relation to the semiannual oscillation of the zonal wind. J. Atmos. Sci., 35, 714-722.

Holton, J. R., 1972: Waves in the equatorial stratosphere generated by tropospheric heat sources. J. Atmos. Sci., 29, 368-375.

—, and R. S. Lindzen, 1972: An updated theory for the quasibiennal cycle of the tropical stratosphere. J. Atmos. Sci., 29, 1076-1080.

Horinouchi, T., and S. Yoden, 1996: The excitation of transient waves by localized episodic heating in the tropics and their propagation into the middle atmosphere. J. Meteor. Soc. Japan, 74, 189210.

, and — 1998: Wave-mean flow interaction associated with a QBO-like oscillation simulated in a simplified GCM. J. Atmos. Sci., 55, 502-526.

Hurrell, J. W., J. J. Hack, B. A. Boville, D. L. Willimason, and J. T. Kiehl, 1998: The dynamical simulation of the NCAR Community Climate Model Version 3 (CCM3). J. Climate., 11, 1207-1236.

Janowiak, J. E., and P. A. Arkin, 1991: Rainfall variations in the tropics during 1986-1989 as estimated from observations of cloud top temperature. J. Geophys. Res., 96, 3359-3373.

Kiehl, J. T., J. J. Hack, G. B. Bonan, B. A. Boville, B. P. Briegleb, D. L. Williamson, and P. J. Rasch, 1996: Description of NCAR Community Climate Model (CCM3). Tech. Note NCAR/TN$420+$ STR, Boulder, CO, 152 pp.

Liebmann, B., and D. L. Hartmann, 1982: Interannual variation of outgoing IR associated with the tropical circulation changes during 1974-78. J. Atmos. Sci., 39, 1153-1162. 
Lindzen, R. S., and J. R. Holton, 1968: A theory of the quasi-biennal oscillation. J. Atmos. Sci., 25, 1095-1107.

Longuet-Higgins, M., 1968: The eigenfunctions of Laplace's tidal equation over a sphere. Philos. Trans. Roy. Soc. London, 262A, 511-607.

Madden, R. A., and P. R. Julian, 1994: Observations of the 40-50 day tropical oscillation-A review. Mon. Wea. Rev., 122, 814837.

Manzini, E., and K. Hamilton, 1993: Middle atmosphere traveling waves forced by latent heat and convective heating. J. Atmos. Sci., 50, 2180-2200.

Naujokat, B., 1986: An update of the observed quasi-biennal oscillation of the stratospheric winds over the Tropics. J. Atmos. Sci., 43, 1873-1877.

Plumb, R. A., 1977: The interaction of two internal waves with the mean flow: Implications for the theory of the quasi-biennal oscillation. J. Atmos. Sci., 34, 1847-1858.

— , and R. C. Bell, 1982: A model of the quasi-biennal oscillation on an equatorial beta-plane. Quart. J. Roy. Meteor. Soc., 108, 335-352.

Reed, R. J., 1965: The quasi-biennal oscillation of the atmosphere between 30 and $50 \mathrm{~km}$ over Ascension Island. J. Atmos. Sci., 22, 331-333.

- W. J. Cambell, L. A. Rasmussen, and D. G. Rogers, 1961: Evidence of the downward propagation annual wind reversal in the equatorial stratosphere. J. Geophys. Res., 66, 813-818.

Richards, F., and P. A. Arkin, 1981: On the relationship between satellite-observed cloud cover and precipitation. Mon. Wea. Rev., 109, 1081-1093.

Rossow, W. B., 1989: Measuring cloud properties from space: A review. J. Climate, 2, 201-213

Salby, M. L., and R. R. Garcia, 1987: Transient response to localized episodic heating in the Tropics. Part I: Excitation and short-time near-field behavior. J. Atmos. Sci., 44, 458-498.

- H. H. Hendon, K. Woodberry, and K. Tanaka, 1991: Analysis of global cloud imagery from multiple satellites. Bull. Amer. Meteor. Soc., 72, 467-480.

Sassi, F., and R. R. Garcia, 1997: The role of equatorial waves forced by convection in the tropical semiannual oscillation. J. Atmos. Sci., 54, 1925-1942.

$\longrightarrow,-$, and B. A. Boville, 1993: The stratopause semiannual oscillation in the NCAR Community Climate Model. J. Atmos. Sci., 50, 3608-3624.

Sato, K., and T. J. Dunkerton, 1997: Estimates of momentum flux associated with Kelvin and gravity waves. J. Geophys. Res., 102, $26247-26262$.

— - F. Hasegawa, and I. Hirota, 1994: Short period disturbances in the equatorial lower stratosphere. J. Meteor. Soc. Japan, 72, 859-872.

Schiffer, R. A., and W. B. Rossow, 1983: The International Satellite Cloud Climatology Project (ISCCP): The first project of the World Climate Research Programme. Bull. Amer. Meteor. Soc., 64, 779-784

Slingo, J. M., and Coauthors, 1995: Intraseasonal oscillations in 15 atmospheric General Circulation Models (results from an AMIP diagnostic subproject). World Meteorological Organization, WCRP-88, Geneva, Switzerland, $32 \mathrm{pp}$.

Takahashi, M., 1987: A two dimensional numerical model of the quasi-biennal oscillation: Part I. J. Meteor. Soc. Japan, 65, 523 536

— the equatorial quasi-biennal oscillation. J. Atmos. Sci., 49, 10201035 .

Tanaka, K. H., K. Woodberry, H. H. Hendon, and M. L. Salby, 1991: Assimilation of global cloud imagery from multiple satellites. J. Atmos. Oceanic Technol., 8, 613-626.

Tsuda, T., Y. Murayama, H. Wiryosumarto, S. W. B. Harijono, and S. Kato, 1994a: Radiosonde observations of equatorial atmosphere dynamics over Indonesia. Part I: Equatorial waves and diurnal tides. J. Geophys. Res., 99, 10 491-10 505.

$\leftarrow,-,-,-$, and,$- 1994 \mathrm{~b}$ : Radiosonde observations of equatorial atmosphere dynamics over Indonesia. Part II: Characteristics of gravity waves. J. Geophys. Res., 99, $10507-$ 10516.

Zhang, G. J., and N. A. McFarlane, 1995: Sensitivity of climate simulations to the parameterization of cumulus convection in the Canadian Centre General Circulation Model. Atmos.-Ocean, 33, 407-446.

— , J. T. Kiehl, and P. J. Rasch, 1998: Response of climate simulation to a new convective parameterization in the National Center for Atmospheric Research Community Climate Model (CCM3). J. Climate, 11, 2097-2115. 